\title{
The use of self-management strategies for problem gambling: a scoping review
}

\author{
Flora I. Matheson ${ }^{1 *}$ (D), Sarah Hamilton-Wright ${ }^{1}$, David T. Kryszajtys' ${ }^{1}$, Jessica L. Wiese', Lauren Cadel ${ }^{3}$, Carolyn Ziegler²,
} Stephen W. Hwang ${ }^{1}$ and Sara J. T. Guilcher ${ }^{1,3}$

\begin{abstract}
Background: Problem gambling (PG) is a serious public health concern that disproportionately affects people experiencing poverty, homelessness, and multimorbidity including mental health and substance use concerns. Little research has focused on self-help and self-management in gambling recovery, despite evidence that a substantial number of people do not seek formal treatment. This study explored the literature on PG self-management strategies. Self-management was defined as the capacity to manage symptoms, the intervention, health consequences and altered lifestyle that accompanies a chronic health concern.

Methods: We searched 10 databases to identity interdisciplinary articles from the social sciences, allied health professions, nursing and psychology, between 2000 and June 28, 2017. We reviewed records for eligibility and extracted data from relevant articles. Studies were included in the review if they examined PG self-management strategies used by adults (18+) in at least a subset of the sample, and in which PG was confirmed using a validated diagnostic or screening tool.

Results: We conducted a scoping review of studies from 2000 to 2017, identifying 31 articles that met the criteria for full text review from a search strategy that yielded 2662 potential articles. The majority of studies examined selfexclusion (39\%), followed by use of workbooks (35\%), and money or time limiting strategies (17\%). The remaining $8 \%$ focused on cognitive, behavioural and coping strategies, stress management, and mindfulness.

Conclusions: Given that a minority of people with gambling concerns seek treatment, that stigma is an enormous barrier to care, and that PG services are scarce and most do not address multimorbidity, it is important to examine the personal self-management of gambling as an alternative to formalized treatment.
\end{abstract}

Keywords: Self-management, Gambling, Problem gambling, Self-efficacy, Self-help, Coping, Strategies, Coping skills, Self-exclusion, Gamblers, Scoping review

\section{Background}

Problem gambling (PG) is a serious chronic health condition and public health concern that affects between 0.12 and $5.8 \%$ of the general population worldwide [1], and up to $7 \%$ in some studies [2]. Those who are most susceptible to PG often experience other complex health and social concerns such as homelessness, mental health issues, substance use disorders, and incarceration [3-5]. Existing services are often not integrated and thus are not designed to address concurrent concerns with PG

\footnotetext{
*Correspondence: mathesonf@smh.ca; MathesonF@smh.ca

'MAP Centre for Urban Health Solutions, St. Michael's Hospital, 30 Bond

Street, Toronto, ON M5B 1W8, Canada

Full list of author information is available at the end of the article
}

[6]. Among treatment seeking individuals, there is a need to increase awareness of existing PG-related services and supports [6]. Further, while there are PG interventions that have demonstrated effectiveness, they can be inaccessible to many vulnerable groups due to barriers such as geographical distance, long waitlists, and treatment costs [6-12]. Barriers to treatment of PG are reflected in low rates of treatment-seeking, as some research has found that only 1 in 10 people experiencing PG seek treatment compared to 1 in 5 people with alcohol-related disorders [13, 14]. There are also important individual barriers to help-seeking that must be considered. Factors such as problem denial, a fear of stigmatization, the belief in the normalization of

(c) The Author(s). 2019 Open Access This article is distributed under the terms of the Creative Commons Attribution 4.0 International License (http://creativecommons.org/licenses/by/4.0/), which permits unrestricted use, distribution, and reproduction in any medium, provided you give appropriate credit to the original author(s) and the source, provide a link to the Creative Commons license, and indicate if changes were made. The Creative Commons Public Domain Dedication waiver (http://creativecommons.org/publicdomain/zero/1.0/) applies to the data made available in this article, unless otherwise stated. 
gambling, and the belief that gambling is not a disease are cited as reasons why many do not seek formal treatment [12, 15-18]. Several qualitative studies found that emotions such as pride and shame discourage help-seeking [19-21]. In particular, some individuals felt a sense of shame in admitting their problem in a group context, and feared additional stigmatization when disclosing their struggles to strangers [17]. Overcoming individual barriers related to cognitions and beliefs about gambling is necessary before a person can meaningfully commence help-seeking behaviour, whether it be seeking formal treatment services or making the decision to engage in self-management. Increased public education and awareness of PG symptomology and treatment plays an important role in reducing shame, stigma and denial in individuals [18]. Likewise, self-management may be an attractive alternative to formalized treatment for individuals concerned with shame and the perceptions of others.

To help address these barriers, self-management may be used as an adjunct or as an alternative to treatment, and may also be effective for people experiencing complex needs such as those with PG [22]. Self-management is defined as, "an individual's ability to manage the symptoms, treatment, physical and psychosocial consequences and lifestyle changes inherent in living with a chronic condition. Efficacious self-management encompasses the ability to monitor one's condition and to affect the cognitive, behavioural and emotional responses necessary to maintain a satisfactory quality of life" (p. 178) [23]. Self-management interventions are rooted in improving an individual's self-efficacy to manage symptoms through mastery of skills such as problem solving, decision making, resource utilization, forming a patient/health care provider partnership [24, 25]), modeling, interpreting physical symptoms, and social persuasion [25]. Self-management interventions can be provided individually or in a group setting, and can be facilitated by technology in either context [26, 27].

There is extensive empirical evidence for self-management strategies for a range of chronic health issues [22]. Some preliminary research suggests that self-management interventions may be useful for treating addictions to alcohol [28-30] and cannabis [31]. A review found that self-administered treatments (e.g., self-help book) are effective for treating mild alcohol abuse while more severe cases show better outcomes with the use of therapist mediated treatments [32]. Self-management treatments have been used to manage behavioural issues such as nail-biting, poor physical activity, poor diet and excessive internet use [33]. Whiteman et al. [34] conducted a meta-analysis of programs that teach self-management training (e.g., interpersonal skills, trigger identification) and found that the training is effective for dealing with co-occurring mental and physical health issues such as bipolar disorder and asthma. Despite the effectiveness, there is some evidence that self-help treatments may not be well-suited for individuals experiencing severe psychological problems (e.g., personality disorders) and significant interpersonal difficulties [32]. In these more severe cases where individuals may lack capacity, clinician-administered treatments may be bettered suited.

Despite the promising evidence that self-management strategies can be effective for persons with chronic health concerns and complex needs, reviews exploring the current state of the literature on a wide range of self-management strategies for PG are limited. For instance, Raylu et al. [35] reviewed self-help treatment studies up to the year 2008 and found that research on these treatments for PG was still in its infancy. The researchers noted that most studies focused on only two strategies (i.e., self-help manuals and audiotapes), and discussed the importance of exploring a wider range of self-help strategies. In line with this work, Rodda et al. [36] identified six change strategies described by online counselling session clients, and in later examined the perceived helpfulness of 15 cognitive change strategies, noting differences in the helpfulness of particular strategies based on gambling severity as well as age [37]. Although the authors provide an extensive list of strategies, they acknowledge that some strategies may have been missed or conceptualized differently than in past literature. The objective of this scoping review was to build on this work and identify and describe what was reported in the literature on PG self-management strategies.

\section{Methods}

\section{Criteria for inclusion and exclusion}

The basis for the methodology of this scoping review is the five-stage approach suggested by Arksey and O'Malley: (1) identify the research question, (2) identify relevant studies, (3) select relevant studies, (4) chart the data, (5) collate, summarize and report the results [38]. We followed the guidelines of the PRISMA-P [39] as the PRISMA-ScR [40] was not available during the review process. We completed the PRISMA-ScR as a Additional file 1 document to this paper. We did not provide a critical assessment of the quality of the evidence as this is a developing area of research.

Studies were included if the authors examined PG self-management strategies used by adults (18+) in at least a subset of the sample; and PG was confirmed using a validated diagnostic or screening tool. We defined self-management strategies as techniques used to self-manage gambling activities independently of clinician support. The independent use of a strategy 
includes use after a therapy session, use after being introduced by a researcher, and use outside of interactions with researchers and therapists. Some examples of self-management strategies that fit this definition include money limiting strategies, self-management components of Cognitive Behavioural Therapy (CBT; e.g., workbooks, thought records, journaling), coping strategies, and mindfulness. We included randomized controlled trials, observational (cohort, cross-sectional, case-control), descriptive, qualitative, and mixed methods studies. We examined systematic, scoping, realist, and narrative reviews to identify additional studies that met our inclusion criteria. Studies were excluded if they only included face-to-face treatment without a self-management component, peer support groups such as Gambler's Anonymous or online discussion forums, strategies that focused only on gaining knowledge and awareness, and studies examining treatment-seeking behaviour. We also excluded non-peer reviewed works such as reports, theses, dissertations, conference presentations, conference papers, books, book reviews, case studies, trial papers and protocols.

\section{Search strategy}

We collected studies for our review using a search strategy developed by an information specialist and the project team (see Additional file 2 for the full MEDLINE strategy). The following databases were searched in June 2017: Medline, PsycINFO, Embase, the Cochrane Library, CINAHL, Applied Social Sciences Index \& Abstracts, International Bibliography of the Social Sciences, ProQuest Dissertations \& Theses Global, Social Services Abstracts, and Sociological Abstracts.

Our selection of databases ensured interdisciplinary coverage of research in social sciences, allied health professions, nursing and psychology. We used search terms that included a combination of keywords and subject headings for the concepts of gambling and self-management, combined with the Boolean operator "AND." We limited the search to articles published in English or French between 2000 and June 28, 2017. Papers published in French with an English equivalent translation were considered for the review, but none were identified. We supplemented the database searches with cited reference searching. Citations were managed using EndNote.

\section{Study selection}

The next step in the review was to select relevant studies. First, three team members independently reviewed 30 studies to pilot the eligibility criteria for the title and abstract review. Any conflicts were resolved through a larger team discussion. The team refined the inclusion and exclusion criteria based on the pilot and then independently reviewed titles and abstracts of all 2662 studies identified through the search strategy. A total of 169 studies were identified as eligible for full text review. Three team members piloted 17 studies for the full-text review, and then independently reviewed the 169 studies for eligibility and extracted data from 31 articles that met the inclusion criteria. See Fig. 1 for the flowchart of study selection and screening.

\section{Data extraction}

To chart our data, three team members independently extracted information from eligible publications using a data extraction tool the team developed, piloted and modified. The tool provided detailed instructions and formatting guidelines for the data extraction and charting. The following information was extracted using the tool: publication details (authors, publishing year, journal), research objectives, type and description of self-management strategy, methodology, method, outcome measures, sample information and demographics, information on tools used to measure PG, qualitative findings, and authors' main conclusions. For the purposes of this review, self-management strategies were organized into four categories: behavioural self-management $(n=19)$, cognitive self-management $(n=2)$, coping skills/styles $(n=12)$, and multi-part interventions $(n=16)$.

\section{Results}

\section{Description of studies}

Table 1 describes the characteristics of included studies. Most studies were conducted in Canada $(n=11)$, Australia $(n=7)$, and the United States $(n=5)$. Studies were also conducted in Finland, Sweden, Germany, Switzerland, Spain, Greece, Singapore, and New Zealand. The majority of studies included were quantitative $(n=$ $24)$, with fewer being qualitative $(n=3)$ or mixed-methods $(n=4)$. Most studies had a mix of males and females within their samples $(n=27)$; only two studies used an all-male sample and two studies used an all-female sample. The majority of studies $(n=17)$ did not report on race or ethnicity. Of the studies that reported race, most had a majority white sample $(n=11)$. Some studies reported on ethnicities rather than race $(n$ $=6$ ), with samples in which the majority of participants were Canadian $(\mathrm{n}=4)$, Australian $(\mathrm{n}=1)$, and Chinese $(\mathrm{n}=1)$. Three studies were conducted in a clinical setting. One study included participants from a rural setting, and one study included participants with low-income backgrounds. Of the 31 studies, 16 included participants with mental health and/or substance use comorbidities. Health comorbidities with PG included mood disorders (depression, manic depression, bipolar disorder, dysthymia, suicide ideation), substance use disorders (alcoholism, drugs), impulse control disorders 


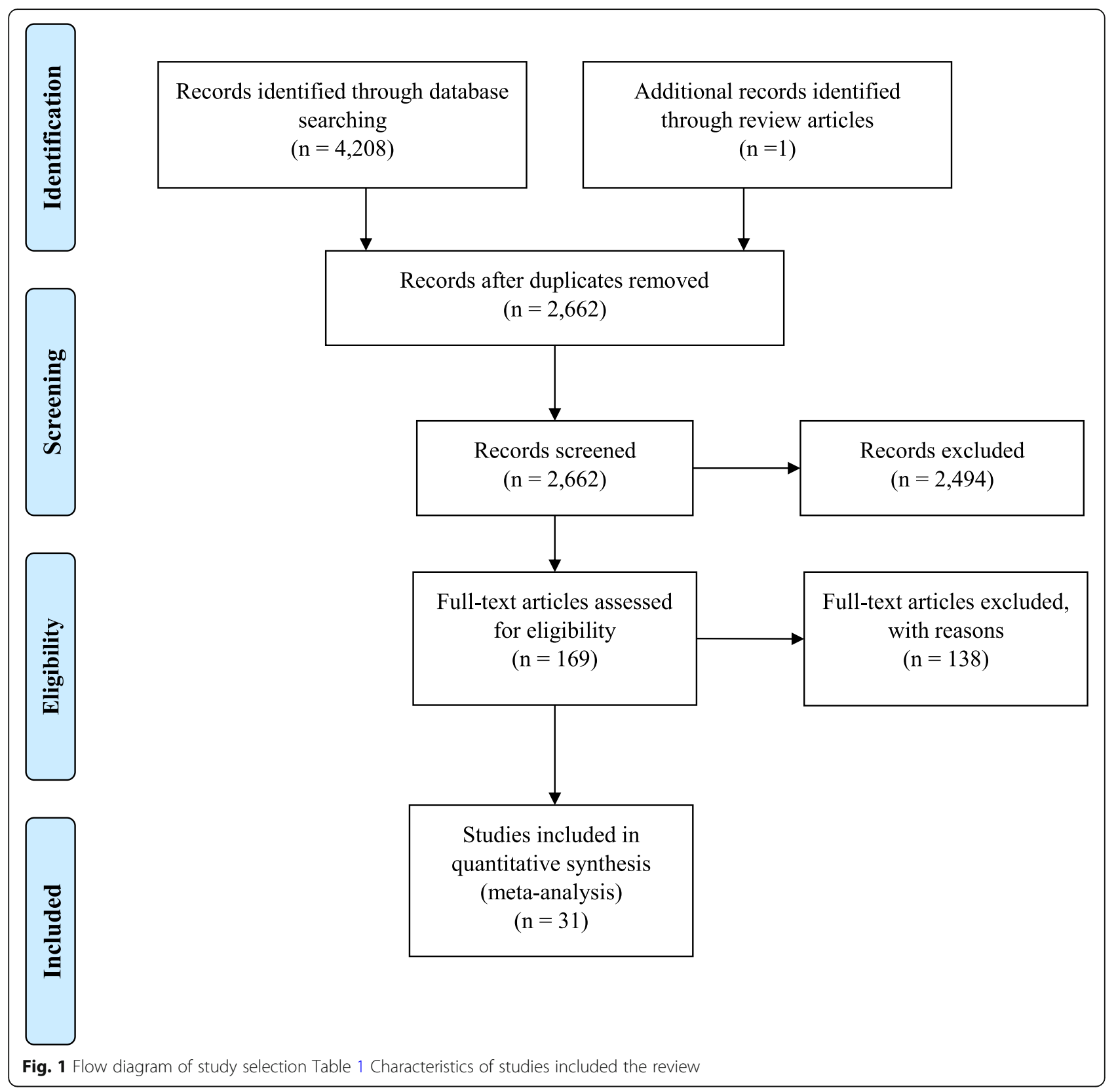

(compulsive buying, compulsive sexual behaviour, kleptomania), anxiety disorders (social phobia, obsessive-compulsive disorder, panic disorder), eating disorders, and experiences of emotional abuse, sexual abuse, physical abuse, loss, stress, and head injury.

\section{Self-management strategies}

Table 2 provides detailed descriptions and key findings regarding the self-management strategies. From a total of 31 studies, we identified 24 self-management strategies. Most studies examined one strategy $(n=25)$, three included two strategies $[41,64,70]$, three included three or more strategies $[59,67,69]$.

\section{Behavioural strategies}

Behavioural self-management strategies are those in which people modify an aspect of their behaviour in order to manage their gambling. Strategies included in this category were self-exclusion $[41,49,50,52$, $53,61,62,68,71]$, money and time limiting [51, 52, $63,67,69]$, alternative activity scheduling [41], direct action [67], social experience [67], delayed gratification [69] and maintenance of balance [69]. While definitions of self-exclusion varied across studies, it was generally defined as entering into formal agreement with a land-based or online gambling venue to be excluded from the venue. In most cases, the 
Table 1 Characteristics of studies included the review

\begin{tabular}{|c|c|c|c|c|c|c|c|}
\hline $\begin{array}{l}\text { Authors; } \\
\text { Country }\end{array}$ & Method & $N$, sex & $\begin{array}{l}\text { Age in years M (SD), } \\
\text { Range }^{a}\end{array}$ & Race/ Ethnicity & $\begin{array}{l}\text { Clinical } \\
\text { Setting } \\
(\mathrm{Y} / \mathrm{N})^{\mathrm{b}}\end{array}$ & $\begin{array}{l}\text { Special } \\
\text { Population }^{c}\end{array}$ & $\begin{array}{l}\text { Health Comorbidities with } \\
\text { Problem Gambling }\end{array}$ \\
\hline $\begin{array}{l}\text { Avery et al. } \\
\text { (2008) [41]; } \\
\text { Not reported }\end{array}$ & Mixed & $\begin{array}{l}N=136 \\
100 \% \text { female }\end{array}$ & $\begin{array}{l}18-24(2.2 \%) ; 25-34 \\
(15.4 \%) ; 35-44 \\
(28.7 \%) ; 45-54 \\
(35.3 \%) ; 55-64 \\
(11.8 \%) ; 65+(3.7 \%) ; \\
\text { Missing (2.9\%) }\end{array}$ & $\begin{array}{l}\text { White (91.9\%), African- } \\
\text { American (2.9\%), Asian } \\
\text { American (2.2\%), Hispanic } \\
(0.7 \%) \text {, Other (1.5\%), Missing } \\
(0.7 \%)\end{array}$ & No & No & $\begin{array}{l}\text { Mental illness (depression, } \\
\text { bipolar disorder and } \\
\text { dysthymia), drug addiction, } \\
\text { alcoholism }\end{array}$ \\
\hline $\begin{array}{l}\text { Boughton et } \\
\text { al. (2016) [42]; } \\
\text { Canada }\end{array}$ & Mixed & $\begin{array}{l}N=25 ; 100 \% \\
\text { female }\end{array}$ & 56.0 & White (85\%) & No & No & $\begin{array}{l}\text { Emotional abuse, sexual abuse, } \\
\text { physical abuse, experiences of } \\
\text { loss, trauma, mental health } \\
\text { issues (depression, anxiety, } \\
\text { panic, manic depression, } \\
\text { anger), suicide ideation }\end{array}$ \\
\hline $\begin{array}{l}\text { Campos et al. } \\
\text { (2016) [43]; } \\
\text { United States }^{d}\end{array}$ & Quant. & $\begin{array}{l}N=87 ; 76 \% \\
\text { male, } 24 \% \\
\text { female }\end{array}$ & 43.4 (10.8); 45.1 (11.0) & $\begin{array}{l}\text { White (44\%), African } \\
\text { American (32\%), Asian (12\%), } \\
\text { Other (12\%) }\end{array}$ & No & $\begin{array}{l}\text { Low } \\
\text { income }\end{array}$ & $\begin{array}{l}\text { Generalized anxiety disorder, } \\
\text { major depressive disorder, } \\
\text { dysthymia, psychotic disorders, } \\
\text { alcohol and/ or marijuana } \\
\text { abuse/ dependence }\end{array}$ \\
\hline $\begin{array}{l}\text { Casey et al. } \\
\text { (2017) [44]; } \\
\text { Australia }^{\text {e }}\end{array}$ & Quant. & $\begin{array}{l}N=174 ; 41 \% \\
\text { male, } 59 \% \\
\text { female }\end{array}$ & $\begin{array}{l}44.8(9.0) ; 44.1(10.5) ; \\
44.2(9.5)\end{array}$ & $\begin{array}{l}\text { White }(81 \%) \text {, Asian }(2 \%), \\
\text { Other }(17 \%) \text {; White }(76 \%) \text {, } \\
\text { Asian }(6 \%) \text {, Other }(18 \%) ; \\
\text { White }(82 \%) \text {, Asian }(2 \%), \\
\text { Other }(16 \%)\end{array}$ & No & No & $\begin{array}{l}\text { Depression, anxiety and stress, } \\
\text { alcohol use }\end{array}$ \\
\hline $\begin{array}{l}\text { Castren et al. } \\
\text { (2013) [45]; } \\
\text { Finland }\end{array}$ & Quant. & $\begin{array}{l}N=471 ; 69 \% \\
\text { male, } 31 \% \\
\text { females }\end{array}$ & $34.5(11.8)$ & Not reported & Yes & No & $\begin{array}{l}\text { Alcohol consumption, } \\
\text { depression }\end{array}$ \\
\hline $\begin{array}{l}\text { Cunningham } \\
\text { et al. (2012) } \\
\text { [46]; Canada }\end{array}$ & Quant. & $\begin{array}{l}N=176^{\mathrm{f}} \\
51 \% \text { male, } \\
49 \% \text { female }\end{array}$ & $47.6(13.4)$ & Not reported & No & No & Not reported \\
\hline $\begin{array}{l}\text { Forsstrom et } \\
\text { al. (2017) [47]; } \\
\text { Sweden }\end{array}$ & Qual. & $\begin{array}{l}N=20 ; 95 \% \\
\text { male, } 5 \% \\
\text { female }\end{array}$ & $42.15(12.70)$ & Not reported & No & No & Not reported \\
\hline $\begin{array}{l}\text { Grant et al. } \\
\text { (2011) [48]; } \\
\text { United States }\end{array}$ & Quant. & $\begin{array}{l}N=68(35 \\
\text { follow-up); } \\
62 \% \text { male, } \\
38 \% \text { female }\end{array}$ & $49.55 ; 48.51$ & White (94\%) & No & No & $\begin{array}{l}\text { Mood disorder (major } \\
\text { depressive disorder, dysthymia, } \\
\text { depressive disorder not } \\
\text { otherwise specified), impulse } \\
\text { control disorder (compulsive } \\
\text { buying, compulsive sexual } \\
\text { behaviour, or kleptomania), } \\
\text { anxiety disorder (social phobia, } \\
\text { obsessive-compulsive disorder, } \\
\text { panic disorder), eating disorder }\end{array}$ \\
\hline $\begin{array}{l}\text { Hayer and } \\
\text { Meyer (2011) } \\
\text { [49]; Australia, } \\
\text { Germany, } \\
\text { Switzerland }\end{array}$ & Quant. & $\begin{array}{l}N=152 ; 72 \% \\
\text { male; } 28 \% \\
\text { female }\end{array}$ & 41.3 & Not reported & No & No & Not reported \\
\hline $\begin{array}{l}\text { Hing, Russell, } \\
\text { Gainsbury et } \\
\text { al. (2015) [50]; } \\
\text { Australia }\end{array}$ & Quant. & $\begin{array}{l}N=620 ; 80 \% \\
\text { male, } 20 \% \\
\text { female }\end{array}$ & $37.6(13.1)$ & Not reported & No & No & Psychological distress \\
\hline $\begin{array}{l}\text { Hing, } \\
\text { Sproston et al. } \\
\text { (2017) [51]; } \\
\text { Australia }\end{array}$ & Quant. & $\begin{array}{l}N=860 ; 68 \% \\
\text { male, } 32 \% \\
\text { female }\end{array}$ & $49.5(15.9)$ & Not reported & No & No & Not reported \\
\hline $\begin{array}{l}\text { Hing, } \\
\text { Cherney, et al. } \\
\text { (2015) [52]; } \\
\text { Australia }\end{array}$ & Qual. & $\begin{array}{l}N=25 ; 100 \% \\
\text { male }\end{array}$ & $39.9(14.1)$ & Not reported & No & No & Not reported \\
\hline $\begin{array}{l}\text { Hing, Russell, } \\
\text { Tolchard, et al. }\end{array}$ & Quant. & $\begin{array}{l}N=103 ; 56 \% \\
\text { male, } 44 \%\end{array}$ & $66.2(6.5)$ & Not reported & No & No & Alcoholism \\
\hline
\end{tabular}


Table 1 Characteristics of studies included the review (Continued)

\begin{tabular}{|c|c|c|c|c|c|c|c|}
\hline $\begin{array}{l}\text { Authors; } \\
\text { Country }\end{array}$ & Method & $N$, sex & $\begin{array}{l}\text { Age in years } M(S D), \\
\text { Range }^{a}\end{array}$ & Race/ Ethnicity & $\begin{array}{l}\text { Clinical } \\
\text { Setting } \\
(\mathrm{Y} / \mathrm{N})^{\mathrm{b}}\end{array}$ & $\begin{array}{l}\text { Special } \\
\text { Population }^{c}\end{array}$ & $\begin{array}{l}\text { Health Comorbidities with } \\
\text { Problem Gambling }\end{array}$ \\
\hline $\begin{array}{l}\text { (2015) [53]; } \\
\text { Australia }\end{array}$ & & female & & & & & \\
\hline $\begin{array}{l}\text { Hodgins } \\
\text { (2005) [54]; } \\
\text { Canada }\end{array}$ & Quant. & $\begin{array}{l}N=102 ; 48 \% \\
\text { male, } 52 \% \\
\text { female }\end{array}$ & $46(9)$ & $\begin{array}{l}\text { Canadian (89\%), Native (2\%), } \\
\text { Other (7\%) }\end{array}$ & No & No & Not reported \\
\hline $\begin{array}{l}\text { Hodgins et al. } \\
\text { (2009) [55]; } \\
\text { Canada }\end{array}$ & Quant. & $\begin{array}{l}N=314 ; 45 \% \\
\text { male, } 55 \% \\
\text { female }\end{array}$ & Not reported & Not reported & No & No & $\begin{array}{l}\text { Mental health disorders, alcoho } \\
\text { abuse or dependence, major } \\
\text { depressive disorder, bipolar } \\
\text { disorder (lifetime) }\end{array}$ \\
\hline $\begin{array}{l}\text { Hodgins et al. } \\
\text { (2001) [56]; } \\
\text { Canada }\end{array}$ & Quant. & $\begin{array}{l}\mathrm{N}=102 ; 48 \% \\
\text { male, } 52 \% \\
\text { female }\end{array}$ & $46(9)$ & $\begin{array}{l}\text { Canadian (89\%), Native (2\%), } \\
\text { Other (7\%) }\end{array}$ & No & No & Not reported \\
\hline $\begin{array}{l}\text { Hodgins et al. } \\
\text { (2007) [57]; } \\
\text { Canada }\end{array}$ & Quant. & $\begin{array}{l}N=169 ; 58 \% \\
\text { male, } 42 \% \\
\text { female }\end{array}$ & $32(11.2)$ & $\begin{array}{l}\text { Canadian (89\%), Other (7\%), } \\
\text { Native/ Metis (4\%) }\end{array}$ & No & No & $\begin{array}{l}\text { Depression, alcohol or drug } \\
\text { addiction, emotional/ mental } \\
\text { health difficulties }\end{array}$ \\
\hline $\begin{array}{l}\text { Hodgins, } \\
\text { Currie, el- } \\
\text { Guebaly, } \\
\text { Peden et al. } \\
\text { (2004) [58]; } \\
\text { Canadah }\end{array}$ & Quant. & $\begin{array}{l}\mathrm{N}=102(52 \\
\text { follow-up); } \\
44 \% \text { male, } \\
56 \% \text { female }\end{array}$ & $46.0(10.0)$ & $\begin{array}{l}\text { English Canadian (90\%), } \\
\text { Aboriginal (2\%), French } \\
\text { Canadian (2\%), Other (6\%) }\end{array}$ & No & No & Not reported \\
\hline $\begin{array}{l}\text { Jauregui et al. } \\
\text { (2017) [59]; } \\
\text { Spain }\end{array}$ & Quant. & $\begin{array}{l}N=274 ; \\
100 \% \text { male }\end{array}$ & 39.3 (11.8); 33.4 (11.9) & Not reported & Yes & No & Depression, anxiety \\
\hline $\begin{array}{l}\text { Labrie et al. } \\
\text { (2012) [60]; } \\
\text { United States }\end{array}$ & Quant. & $\begin{array}{l}N=248 ; 52 \% \\
\text { male, } 48 \% \\
\text { female; } 66 \% \\
\text { male, } 34 \% \\
\text { female }\end{array}$ & $44.0(14.0) ; 49.0(10.0)$ & $\begin{array}{l}\text { White }(74 \%), \text { Hispanic }(15 \%) \text {; } \\
\text { White }(66 \%), \text { Hispanic }(3 \%)\end{array}$ & No & Rural & $\begin{array}{l}\text { Mental/ emotional problems, } \\
\text { drug/ alcohol problems }\end{array}$ \\
\hline $\begin{array}{l}\text { Ladouceur et } \\
\text { al. (2000) [61]; } \\
\text { Canada }\end{array}$ & Quant. & $\begin{array}{l}N=220 ; 62 \% \\
\text { male, } 38 \% \\
\text { female }\end{array}$ & 41.0 & Not reported & No & No & Not reported \\
\hline $\begin{array}{l}\text { Ladouceur et } \\
\text { al. (2007) [62]; } \\
\text { Canada }\end{array}$ & Quant. & $\begin{array}{l}N=161 ; 60 \% \\
\text { males; } 40 \% \\
\text { females }\end{array}$ & $43.5(12.3)$ & Not reported & No & No & Not reported \\
\hline $\begin{array}{l}\text { Lalande and } \\
\text { Ladouceur } \\
\text { (2011) [63]; } \\
\text { Canadak }^{k}\end{array}$ & Mixed & $\begin{array}{l}\mathrm{N}=20 ; \mathrm{N}= \\
65 ; 67 \% \\
\text { male, } 33 \% \\
\text { female; } 66 \% \\
\text { male, } 34 \% \\
\text { female }\end{array}$ & $47.7(13.4) ; 43.6(15.2)$ & Not reported & No & $\begin{array}{l}\text { Low- } \\
\text { income }\end{array}$ & Not reported \\
\hline $\begin{array}{l}\text { Linardatou et } \\
\text { al. (2014) [64]; } \\
\text { Greece }\end{array}$ & Mixed & $\begin{array}{l}N=45 ; \\
95.5 \% \text { male; } \\
4.5 \% \text { female; } \\
90 \% \text { male; } \\
10 \% \text { female }\end{array}$ & 42.6 & Not reported & No & No & Depression, anxiety, stress \\
\hline $\begin{array}{l}\text { Luquiens et al. } \\
\text { (2016) [65]; } \\
\text { Not reported }\end{array}$ & Quant. & $\begin{array}{l}N=1122 ; \\
92 \% \text { male; } \\
8 \% \text { female }\end{array}$ & 34.7 & Not reported & No & No & Not reported \\
\hline $\begin{array}{l}\text { Martin (2013) } \\
\text { [66]; United } \\
\text { States }\end{array}$ & Quant. & $\begin{array}{l}N=60 ; 60 \% \\
\text { male; } 40 \% \\
\text { female }\end{array}$ & $\begin{array}{l}\text { Underclassmen } \\
\text { (Freshman, } \\
\text { Sophomore) (88.3\%); } \\
\text { Upperclassmen } \\
\text { (Junior/ Senior) } \\
(11.7 \%)\end{array}$ & $\begin{array}{l}\text { Caucasian (non-Hispanic) } \\
\text { (71.7\%), African American } \\
\text { (20\%); Hispanic or Latino } \\
(5 \%) \text {, American Indian or } \\
\text { Alaskan Native (1.7\%), Other } \\
(1.7 \%)\end{array}$ & No & No & Not reported \\
\hline $\begin{array}{l}\text { Moore et al. } \\
\text { (2012) [67]; } \\
\text { Australia }\end{array}$ & Quant. & $\begin{array}{l}N=303 ; 39 \% \\
\text { male, } 61 \% \\
\text { female }\end{array}$ & $26.4(10.1)$ & $\begin{array}{l}\text { Australian (79\%), European } \\
(14 \%) \text {, Asian (7\%) }\end{array}$ & No & No & Not reported \\
\hline
\end{tabular}


Table 1 Characteristics of studies included the review (Continued)

\begin{tabular}{|c|c|c|c|c|c|c|c|}
\hline $\begin{array}{l}\text { Authors; } \\
\text { Country }\end{array}$ & Method & N, sex & $\begin{array}{l}\text { Age in years M (SD), } \\
\text { Range }^{\mathrm{a}}\end{array}$ & Race/ Ethnicity & $\begin{array}{l}\text { Clinical } \\
\text { Setting } \\
(\mathrm{Y} / \mathrm{N})^{\mathrm{b}}\end{array}$ & $\begin{array}{l}\text { Special } \\
\text { Population }^{c}\end{array}$ & $\begin{array}{l}\text { Health Comorbidities with } \\
\text { Problem Gambling }\end{array}$ \\
\hline $\begin{array}{l}\text { Nelson et al. } \\
\text { (2010) [68]; } \\
\text { United States }\end{array}$ & Quant. & $\begin{array}{l}N=113 ; 45 \% \\
\text { male, } 55 \% \\
\text { female }\end{array}$ & $45.0(10.0)$ & $\begin{array}{l}\text { White }(80.5 \%) \text {, Black or } \\
\text { African-American (16.8\%), } \\
\text { Other (2.7\%), Hispanic or La- } \\
\text { tino }(0.9 \%)\end{array}$ & No & No & $\begin{array}{l}\text { Substance use, mental health } \\
\text { problems }\end{array}$ \\
\hline $\begin{array}{l}\text { Subramaniam } \\
\text { et al. (2017) } \\
\text { [69]; } \\
\text { Singapore }\end{array}$ & Qual. & $\begin{array}{l}\mathrm{N}=25 ; 72 \% \\
\text { male, } 28 \% \\
\text { females }\end{array}$ & $66.2(6.5)$ & $\begin{array}{l}\text { Chinese }(64 \%) \text {, Indian (16\%), } \\
\text { Other (12\%), Malay (8\%) }\end{array}$ & No & $\begin{array}{l}\text { Adults }(60 \\
+)\end{array}$ & Not reported \\
\hline $\begin{array}{l}\text { Toneatto et al. } \\
\text { (2014) [70]; } \\
\text { Canada }^{\mathrm{m}}\end{array}$ & Quant. & $\begin{array}{l}N=18 ; \\
44.4 \% \text { male, } \\
55.6 \% \\
\text { female; } \\
66.7 \% \text { male, } \\
33.3 \% \\
\text { female }\end{array}$ & $41.7(11.0) ; 46.6(11.8)$ & Not reported & No & No & Current emotional distress \\
\hline $\begin{array}{l}\text { Townshend } \\
\text { (2007) [71]; } \\
\text { New Zealand }\end{array}$ & Quant. & $\begin{array}{l}N=35 ; 60 \% \\
\text { male; } 40 \% \\
\text { female }\end{array}$ & $18-73$ & Not reported & Yes & No & $\begin{array}{l}\text { Head injury, substance use } \\
\text { disorder, other mental health } \\
\text { disorder }\end{array}$ \\
\hline
\end{tabular}

aalues reported when available

${ }^{b}$ Clinical setting includes participants (full or subset of sample) recruited from treatment centers, in-patient programs, residential treatments or therapist-facilitated out-patient treatment programs

'Special designation includes participants (full or subset of sample) who belong to populations such as veteran, homeless/in poverty, correctional or elderly

${ }^{\mathrm{d}}$ Age information provided by condition (workbook only; workbook plus therapist guidance)

${ }^{\mathrm{e}}$ Age and Race/ Ethnicity information provided by condition (internet-based CBT, internet-based MFS, waitlist, respectively)

'Completed at least one follow-up

${ }^{9}$ Age information provided by condition (imaginal desensitization; Gambler's Anonymous control)

${ }^{\mathrm{h}}$ Same sample as Hodgins et al. (2001)

'Age and Race/ Ethnicity information provided by condition (pathological gambling group; non-pathological gambling group)

'Sex, Age and Race/ Ethnicity information provided by sample (Nevada, US; Massachusetts, US)

${ }^{\mathrm{k}}$ Ns presented by study (Pilot; Main Study); Sex and Age provided by sample (problem gambling subset; non-problem gambling subset)

'Sex presented by condition (intervention; control)

${ }^{\mathrm{m}} \mathrm{Sex}$, Age information presented by condition (Mindfulness plus CBT; waitlist)

terms of the agreement included consequences (e.g., fines, trespassing charges) or restrictions on the collection of winnings (i.e., not allowed to collect winnings) when the agreement was breached. Seven studies examined the effectiveness of self-exclusion for PG and generally reported it to have positive results on its own and in combination with counseling; however, one study reported that over $50 \%$ of participants breached self-exclusion agreements within 6 months [62]. Hayer and Meyer [49] reported on the characteristics of people who self-excluded, noting that financial difficulty was the most cited reason for self-exclusion, and that male and middle-aged individuals were most likely to self-exclude. Hing et al. [50] found that people experiencing PG who were involved in problematic internet gambling were less likely to self-exclude from land-based venues (one-fifth of their gambling behavior) and more likely to self-exclude from online gambling sites than their than their land-based counterparts. One study reported that self-exclusion may be more effective in jurisdictions that frame PG as a public health issue because doing so places responsibility on gambling venues instead of people experiencing PG to enforce the ban [71].
Self-limiting strategies with duration of time and the amount of money were described in five studies. Hing et al. [52] described a process in which an individual deposited monetary amounts to bet at the outset of a gambling episode and stopped gambling once that limit was reached. Two studies reported on the effectiveness of limiting strategies, one noting that these strategies predict non-harmful gambling [53] and another reporting limited success [52]. Some evidence indicates that limiting strategies may not be well suited for severe cases of PG. Lalande and Ladouceur [63] reported that those experiencing pathological gambling and those who did not engage in pathological gambling both use money limiting strategies to avoid overspending; however, people experiencing pathological gambling set higher limits and broke these limits more than those who were not experiencing pathological gambling.

Delayed gratification (i.e., quelling the need for immediate results of a gamble) and maintaining balance (i.e., avoiding excesses in behaviour) were self-management strategies reported by older adults $(60+)$ with gambling problems or probable pathological gambling [69]. Alternative activity scheduling (i.e., scheduling non-gambling activities) was effective in reducing PG scores for some women who combined this activity with self-exclusion [41]. 
Table 2 Self-management strategies described in the included studies

\begin{tabular}{|c|c|c|c|}
\hline Authors & $\begin{array}{l}\text { Self-Management } \\
\text { Strategy }\end{array}$ & $\begin{array}{l}\text { Details/ Description of } \\
\text { Self-Management Strategy }\end{array}$ & $\begin{array}{l}\text { Key Findings and Implementation } \\
\text { Considerations related to the } \\
\text { Self-Management Strategy }\end{array}$ \\
\hline \multirow[t]{2}{*}{ Avery et al. (2008) [41] } & \multirow[t]{2}{*}{$\begin{array}{l}\text { - Alternative activity } \\
\text { scheduling } \\
\text { - Self-exclusion }\end{array}$} & $\begin{array}{l}\text { Alternative Activity Scheduling } \\
\text { - Person plans non-gambling } \\
\text { activities to take the place } \\
\text { of gambling (e.g., joining } \\
\text { social groups, making } \\
\text { plans to fill time) }\end{array}$ & \multirow{2}{*}{$\begin{array}{l}\text { - Gender differences identified for } \\
\text { motivation to stop gambling and } \\
\text { supports sought; most women } \\
\text { used GA, professional help (or both) } \\
\text { - A small proportion (10\%) of women } \\
\text { recovered on their own from PG } \\
\text { using alternative activity scheduling } \\
\text { and self-exclusion and had lower PG } \\
\text { scores than those receiving } \\
\text { professional or GA help }\end{array}$} \\
\hline & & $\begin{array}{l}\text { Self-exclusion } \\
\text { - Person relocates away or } \\
\text { self-bans from gambling } \\
\text { establishments for a } \\
\text { fixed period of time. }\end{array}$ & \\
\hline $\begin{array}{l}\text { Boughton et al. } \\
\text { (2016) [42] }\end{array}$ & $\begin{array}{l}\text { Workbook } \\
\text { (web-based) }\end{array}$ & $\begin{array}{l}\text { - Workbook contains } 12 \\
\text { weekly modules } \\
\text { - Modules cover: change process, } \\
\text { urges and relapses, exploration } \\
\text { of how thoughts impact feelings, } \\
\text { mindfulness, stress management, } \\
\text { relationships, emotional } \\
\text { regulation, goals. }\end{array}$ & $\begin{array}{l}\text { - The workbook was well } \\
\text { received by participants } \\
\text { - Web- and phone-based group } \\
\text { sessions are effective } \\
\text { to expand PG services }\end{array}$ \\
\hline $\begin{array}{l}\text { Campos et al. } \\
\text { (2016) [43] }\end{array}$ & Workbook & $\begin{array}{l}\text { - Person reads and completes } \\
\text { exercises in workbook on } \\
\text { topics such as awareness of } \\
\text { PG behaviour and reasons } \\
\text { for gambling, motivation } \\
\text { for behaviour change, money } \\
\text { limiting, changing faulty } \\
\text { cognitions about gambling, } \\
\text { tools to maintain abstinence. }\end{array}$ & $\begin{array}{l}\text { - Use of self-help workbooks helps } \\
\text { reduce PG symptoms and money } \\
\text { spent gambling, but using a } \\
\text { workbook with therapist } \\
\text { guidance had superior outcomes }\end{array}$ \\
\hline Casey et al. (2017) [44] & $\begin{array}{l}\text { Internet-based } \\
\text { cognitive } \\
\text { behavioural therapy }\end{array}$ & $\begin{array}{l}\text { - Six weeks of CBT sessions } \\
\text { with exercises. } \\
\text { - Sessions cover: awareness } \\
\text { of triggers and strategies to } \\
\text { cope with urges, challenging } \\
\text { thinking errors and replacing } \\
\text { them with helpful thoughts, } \\
\text { debt management, imaginal } \\
\text { desensitization, relaxation } \\
\text { training, problem solving, } \\
\text { goal setting, emotions, } \\
\text { and maintenance and } \\
\text { relapse prevention. }\end{array}$ & $\begin{array}{l}\text { - CBT was associated with reduced } \\
\text { gambling severity, other beneficial } \\
\text { PG and mental health outcomes, } \\
\text { and greater satisfaction after initial } \\
\text { treatment and 12-month follow-up } \\
\text { - Online treatments for gambling } \\
\text { may be a valuable tool in increasing } \\
\text { help-seeking and treatment engagement }\end{array}$ \\
\hline Castren et al. (2013) [45] & $\begin{array}{l}\text { Internet-based } \\
\text { cognitive } \\
\text { behavioural therapy }\end{array}$ & $\begin{array}{l}\text { - } 8 \text { weeks of CBT program } \\
\text { with weekly module. } \\
\text { - Each CBT module contains } \\
\text { information, exercises and } \\
\text { homework covering: } \\
\text { Psychoeducation and motivation, } \\
\text { recognizing high risk situations } \\
\text { and triggers, identifying social } \\
\text { consequences of gambling, } \\
\text { recognizing erroneous thoughts, } \\
\text { safe ways to manage high-risk } \\
\text { situations, money management. }\end{array}$ & $\begin{array}{l}\text { - CBT was associated with reduced } \\
\text { gambling-related problems, urges, } \\
\text { impaired control of gambling, } \\
\text { alcohol consumption, social } \\
\text { consequences, gambling-related } \\
\text { cognitive erroneous } \\
\text { thoughts and depression }\end{array}$ \\
\hline $\begin{array}{l}\text { Cunningham et al. } \\
\text { (2012) [46] }\end{array}$ & $\begin{array}{l}\text { Personalized } \\
\text { feedback tool }\end{array}$ & $\begin{array}{l}\text { - Person completes Problem } \\
\text { Gambling Severity Index (PGSI) } \\
\text { and Gambling Cognitions } \\
\text { Questionnaire. } \\
\text { - Feedback tool provides a } \\
\text { summary of the individual's } \\
\text { PGSI scores and summary of } \\
\text { cognitive distortions that they } \\
\text { endorsed (along with summary } \\
\text { of the error of each of these beliefs); } \\
\text { a list of techniques to lower } \\
\text { gambling risks; a comparison } \\
\text { of the amount of money the } \\
\text { individual spent in the past } \\
\text { year with the average amount } \\
\text { of money spent by Canadians } \\
\text { of the same sex. }\end{array}$ & $\begin{array}{l}\text { - No evidence for the impact of } \\
\text { normative personalized feedback; } \\
\text { however, participants who received } \\
\text { partial feedback (without norms) } \\
\text { reduced the number of days } \\
\text { gambled compared to those } \\
\text { not receiving the intervention } \\
\text { - Personalized feedback interventions } \\
\text { were well received and Internet-based } \\
\text { personalized feedback tools may } \\
\text { improve access to interventions }\end{array}$ \\
\hline Forsstrom et al. (2017) & Personalized & - Individual completes a weekly & - Participants had a positive view \\
\hline
\end{tabular}


Table 2 Self-management strategies described in the included studies (Continued)

\begin{tabular}{|c|c|c|c|}
\hline Authors & $\begin{array}{l}\text { Self-Management } \\
\text { Strategy }\end{array}$ & $\begin{array}{l}\text { Details/ Description of } \\
\text { Self-Management Strategy }\end{array}$ & $\begin{array}{l}\text { Key Findings and Implementation } \\
\text { Considerations related to the } \\
\text { Self-Management Strategy }\end{array}$ \\
\hline [47] & feedback tool & $\begin{array}{l}\text { risk assessment test. } \\
\text { - Feedback tool provides } \\
\text { a risk level based on } \\
\text { comparison of gambling } \\
\text { patterns with other users, } \\
\text { a detailed history of } \\
\text { gambling habits and advice } \\
\text { about how to limit time } \\
\text { and money spent on gambling. }\end{array}$ & $\begin{array}{l}\text { of the tool's content, which should } \\
\text { have promoted use; however, } \\
\text { repeated use was low } \\
\text { - A lack of feedback from the } \\
\text { tool and confusion when signing } \\
\text { up may have affected usage; } \\
\text { offering users direct } \\
\text { feedback may increase usage }\end{array}$ \\
\hline Grant et al. (2011) [48] & $\begin{array}{l}\text { Imaginal } \\
\text { Desensitization }\end{array}$ & $\begin{array}{l}\text { - } 6 \text { sessions of imaginal } \\
\text { desensitization plus } \\
\text { motivational interviewing } \\
\text { (IDMI) over an 8-week period } \\
\text { - Audio-recordings of three } \\
\text { gambling scenarios are played } \\
\text { three times per day which prompt } \\
\text { the user to use relaxation coping s } \\
\text { trategies to cope with urges } \\
\text { the scenarios elicit. }\end{array}$ & $\begin{array}{l}\text { - The intervention reduced PG } \\
\text { urges and behaviour; effects } \\
\text { were largely maintained for } 6 \text { months } \\
\text { - Participants cited their } \\
\text { audiotapes as the primary } \\
\text { reason for their improvement. }\end{array}$ \\
\hline $\begin{array}{l}\text { Hayer and Meyer (2011) } \\
\text { [49] }\end{array}$ & Self-exclusion & $\begin{array}{l}\text { - Once registered, the individual is } \\
\text { banned from gambling venues } \\
\text { and websites for a fixed period of time. }\end{array}$ & $\begin{array}{l}\text { - Mostly men and middle-aged } \\
\text { individuals place themselves } \\
\text { on exclusion lists; top motives } \\
\text { to self-exclude involve financial difficulties } \\
\text { - Self-exclusion is effective when } \\
\text { used in combination with } \\
\text { additional counseling }\end{array}$ \\
\hline $\begin{array}{l}\text { Hing, Russell, Gainsbury } \\
\text { et al. (2015) [50] }\end{array}$ & Self-exclusion & $\begin{array}{l}\text { - Once registered, the individual is } \\
\text { banned from gambling venues } \\
\text { and websites for a fixed period of time. }\end{array}$ & $\begin{array}{l}\text { - Land-based gamblers are more } \\
\text { likely to use self-exclusion } \\
\text { strategies than problem } \\
\text { Internet gamblers }\end{array}$ \\
\hline $\begin{array}{l}\text { Hing, Sproston et al. } \\
\text { (2017) [51] }\end{array}$ & $\begin{array}{l}\text { Responsible } \\
\text { Gambling Strategies } \\
\text { - Money and } \\
\text { time limiting }\end{array}$ & $\begin{array}{l}\text { - When gambling, Person carries } \\
\text { limited money, stops gambling } \\
\text { once it is spent, does not carry } \\
\text { bank card, does not re-gamble } \\
\text { any wins, and places smaller bets. }\end{array}$ & $\begin{array}{l}\text { - Setting money limits and } \\
\text { balancing gambling with } \\
\text { other activities predict } \\
\text { non-harmful gambling }\end{array}$ \\
\hline \multirow[t]{2}{*}{$\begin{array}{l}\text { Hing, Cherney, et al. } \\
\text { (2015) [52] }\end{array}$} & $\begin{array}{l}\text { Self-exclusion } \\
\text { (online) }\end{array}$ & $\begin{array}{l}\text { - Individual blocks themselves from } \\
\text { specific gambling websites using } \\
\text { website blocking software. }\end{array}$ & \multirow{2}{*}{$\begin{array}{l}\text { - Limiting strategies had variable } \\
\text { success; most felt that operators } \\
\text { needed to implement more } \\
\text { responsible gambling measures } \\
\text { (e.g., removal of credit betting, } \\
\text { imposed bet limits, gambling } \\
\text { help pop-up messages, } \\
\text { restricted promotions/ advertising) }\end{array}$} \\
\hline & $\begin{array}{l}\text { Money limit setting } \\
\text { (online) }\end{array}$ & $\begin{array}{l}\text { - Individual deposits monetary } \\
\text { amounts to bet at the outset } \\
\text { of gambling episode and they } \\
\text { prohibit themselves } \\
\text { from credit betting. }\end{array}$ & \\
\hline $\begin{array}{l}\text { Hing, Russell, Tolchard, } \\
\text { et al. (2015) [53] }\end{array}$ & Self-exclusion & $\begin{array}{l}\text { - Individual excludes themselves } \\
\text { from a range of venue types } \\
\text { (e.g., hotels, clubs and casinos } \\
\text { that operate electronic } \\
\text { gaming machines) }\end{array}$ & $\begin{array}{l}\text { - Self-excluders abstained from } \\
\text { most problematic gambling and fewer had } \\
\text { - harmful consequences vs. non-excluders } \\
\text { - Self-exclusion may have similar } \\
\text { short-term outcomes as } \\
\text { counselling alone and may } \\
\text { reduce short-term harms }\end{array}$ \\
\hline Hodgins $(2005)^{\mathrm{a}}[54]$ & Workbook & $\begin{array}{l}\text { - CBT-based workbook includes } \\
\text { self-assessment, goal setting, } \\
\text { strategies (e.g., self-exclusion, } \\
\text { alternative activity scheduling, } \\
\text { cognitive restructuring), } \\
\text { maintenance and local } \\
\text { treatment resources }\end{array}$ & $\begin{array}{l}\text { - Intervention reduced PG behaviour } \\
\text { and reduction was } \\
\text { maintained at } 12 \text { - and } 24 \text {-months }\end{array}$ \\
\hline $\begin{array}{l}\text { Hodgins et al. (2009) } \\
\text { [55] }\end{array}$ & Workbook & $\begin{array}{l}\text { - CBT workbook includes } \\
\text { self-assessment, goal setting, } \\
\text { strategies (e.g., self-exclusion, } \\
\text { Iternative activity scheduling, } \\
\text { cognitive restructuring), } \\
\text { maintenance and local } \\
\text { treatment resources }\end{array}$ & $\begin{array}{l}\text {-Workbook only group participants } \\
\text { were just as likely to have significantly } \\
\text { reduced their losses over the year } \\
\text { and to not meet criteria for pathological } \\
\text { gambling as those in brief } \\
\text { treatment and brief booster treatment }\end{array}$ \\
\hline $\begin{array}{l}\text { Hodgins et al. (2001) } \\
\text { [56] }\end{array}$ & Workbook & $\begin{array}{l}\text { - CBT workbook includes } \\
\text { self-assessment, goal setting, } \\
\text { strategies (e.g., self-exclusion, } \\
\text { alternative activity scheduling, } \\
\text { cognitive restructuring), }\end{array}$ & $\begin{array}{l}\text { - Participants who received a } \\
\text { motivational enhancement telephone } \\
\text { intervention and a self-help workbook } \\
\text { (vs. workbook only) had better outcomes } \\
\text { than participants in a wait-list control at }\end{array}$ \\
\hline
\end{tabular}


Table 2 Self-management strategies described in the included studies (Continued)

\begin{tabular}{|c|c|c|c|}
\hline Authors & $\begin{array}{l}\text { Self-Management } \\
\text { Strategy }\end{array}$ & $\begin{array}{l}\text { Details/ Description of } \\
\text { Self-Management Strategy }\end{array}$ & $\begin{array}{l}\text { Key Findings and Implementation } \\
\text { Considerations related to the } \\
\text { Self-Management Strategy }\end{array}$ \\
\hline & & $\begin{array}{l}\text { maintenance and local } \\
\text { treatment resources }\end{array}$ & $\begin{array}{l}3 \text { and } 6 \text { month follow-up, but at the } \\
12 \text {-month follow-up, the advantage } \\
\text { of the motivational interview and } \\
\text { workbook condition was found } \\
\text { only for participants with less } \\
\text { severe gambling problems. } \\
\text { Overall, these results support the } \\
\text { effectiveness of a brief telephone } \\
\text { and mail-based treatment } \\
\text { for problem gambling. }\end{array}$ \\
\hline $\begin{array}{l}\text { Hodgins et al. } \\
(2007)[57]\end{array}$ & $\begin{array}{l}\text { Informational } \\
\text { Booklets }\end{array}$ & $\begin{array}{l}\text { - Booklets included topics } \\
\text { on dealing with urges to } \\
\text { gamble; negative emotions } \\
\text { as a cause of relapse; "getting } \\
\text { back on the wagon" after a relapse; } \\
\text { lifestyle balance; financial issues; } \\
\text { stages of change; and dealing } \\
\text { with comorbid emotional } \\
\text { and addiction problems. }\end{array}$ & $\begin{array}{l}\text { - Participants who received } \\
\text { booklet summarizing relapse } \\
\text { prevention information had } \\
\text { improved PG scores, reduced } \\
\text { number of gambling } \\
\text { days and dollars lost }\end{array}$ \\
\hline $\begin{array}{l}\text { Hodgins et al. } \\
\text { (2004) [58] }\end{array}$ & Workbook & $\begin{array}{l}\text { - CBT workbook contains five } \\
\text { sections: (a) self-assessment, } \\
\text { (b) goal setting, (c) strategies, } \\
\text { (d) maintenance, and (e) other } \\
\text { treatment resources. } \\
\text { - Individual completes brief } \\
\text { exercises in each section } \\
\text { of the workbook. }\end{array}$ & $\begin{array}{l}\text { - Participants who received a } \\
\text { motivational telephone } \\
\text { intervention plus a self-help } \\
\text { workbook had better outcomes } \\
\text { (i.e., gambled fewer days, } \\
\text { lost less money, and had } \\
\text { lower South Oaks Gambling } \\
\text { Screen scores) than participants } \\
\text { who received only the workbook }\end{array}$ \\
\hline \multirow[t]{6}{*}{$\begin{array}{l}\text { Jauregui et al. (2017) } \\
\text { [59] }\end{array}$} & \multirow{6}{*}{$\begin{array}{l}\text { Coping Strategies } \\
\text { and Styles } \\
\text { - Problem Solving } \\
\text { - Cognitive } \\
\text { Restructuring } \\
\text { - Social Support } \\
\text { - Emotional } \\
\text { Expression } \\
\text { - Problem Avoidance } \\
\text { - Wishful Thinking } \\
\text { - Social Withdrawal } \\
\text { - Self-Criticism }\end{array}$} & $\begin{array}{l}\text { Problem Solving Strategies: } \\
\text { - Individual focuses on eliminating } \\
\text { stress by modifying the } \\
\text { situation that causes it. } \\
\text { Cognitive Restructuring: } \\
\text { - Individual modifies the meaning } \\
\text { of the stressful situation. }\end{array}$ & \multirow[t]{6}{*}{$\begin{array}{l}\text { - Pathological gamblers (vs non) } \\
\text { obtained significantly higher } \\
\text { scores in pathological gambling, } \\
\text { anxiety, depression, self-criticism, } \\
\text { emotional expression, wishful thinking, } \\
\text { problem avoidance, social withdrawal, } \\
\text { problem disengagement, } \\
\text { emotional disengagement, } \\
\text { and disengagement }\end{array}$} \\
\hline & & $\begin{array}{l}\text { Social Support: } \\
\text { - Individual seeks emotional } \\
\text { support from social circle. } \\
\text { Emotional Expression: } \\
\text { - Individual aims to express the } \\
\text { emotions that arise } \\
\text { in the stress process. }\end{array}$ & \\
\hline & & $\begin{array}{l}\text { Problem Avoidance: } \\
\text { - Individual denies and avoids } \\
\text { thoughts and acts related } \\
\text { to the stressful situation. }\end{array}$ & \\
\hline & & $\begin{array}{l}\text { Wishful Thinking: } \\
\text { • Individual dreams and thinks } \\
\text { about a non-stressful reality. }\end{array}$ & \\
\hline & & $\begin{array}{l}\text { Social Withdrawal: } \\
\text { - Individual distances themselves } \\
\text { from significant people associated } \\
\text { with the emotional reaction } \\
\text { of the stressful situation. }\end{array}$ & \\
\hline & & $\begin{array}{l}\text { Self-criticism: } \\
\text { - Individual engages in self-blame } \\
\text { and self-criticism concerning } \\
\text { the stressful situation itself and } \\
\text { how one dealt with it. }\end{array}$ & \\
\hline Labrie et al. (2012) [60] & Self-help toolkit & $\begin{array}{l}\text { The toolkit provides: } \\
\text { - Exercises such as cost-benefit } \\
\text { analysis of gambling. } \\
\text { - Information about managing } \\
\text { urges, managing change that } \\
\text { comes with dealing with an } \\
\text { addiction, what makes } \\
\text { gambling problematic, } \\
\text { and gambling facts. } \\
\text { - Specific directives about }\end{array}$ & $\begin{array}{l}\text { - Toolkit recipients reported } \\
\text { recently abstaining from gambling } \\
\text { - A self-help toolkit and other } \\
\text { self-directed resources can assist } \\
\text { in remediating gambling-related } \\
\text { problems for individuals who } \\
\text { do not want formal treatment }\end{array}$ \\
\hline
\end{tabular}


Table 2 Self-management strategies described in the included studies (Continued)

\begin{tabular}{|c|c|c|c|}
\hline Authors & $\begin{array}{l}\text { Self-Management } \\
\text { Strategy }\end{array}$ & $\begin{array}{l}\text { Details/ Description of } \\
\text { Self-Management Strategy }\end{array}$ & $\begin{array}{l}\text { Key Findings and Implementation } \\
\text { Considerations related to the } \\
\text { Self-Management Strategy }\end{array}$ \\
\hline & & $\begin{array}{l}\text { how to quit (i.e., skills building). } \\
\text { - Encouragement for people } \\
\text { to practice quitting while } \\
\text { simultaneously preparing } \\
\text { them for failed attempts } \\
\text { (i.e., relapse prevention). }\end{array}$ & \\
\hline $\begin{array}{l}\text { Ladouceur et al. } \\
(2000)[61]\end{array}$ & Self-exclusion & $\begin{array}{l}\text { - Individual excludes themselves } \\
\text { from a government operated } \\
\text { Canadian casino for periods } \\
\text { that range from } \\
6 \text { months to } 5 \text { years. }\end{array}$ & $\begin{array}{l}\text { - } 30 \% \text { of participants ( } 95 \% \text { of } \\
\text { participants were severe } \\
\text { pathological gamblers) reported } \\
\text { that they completely stopped } \\
\text { gambling once enrolled in } \\
\text { the self-exclusion program }\end{array}$ \\
\hline $\begin{array}{l}\text { Ladouceur et al. } \\
\text { (2007) [62] }\end{array}$ & Self-exclusion & $\begin{array}{l}\text { - Three self-exclusion programs } \\
\text { in Quebec Casinos (Montreal, } \\
\text { Gatineau, and Charlevoix), in } \\
\text { which individuals exclude } \\
\text { themselves from casinos } \\
\text { for periods ranging } \\
\text { from } 6 \text { months to } 2 \text { years. }\end{array}$ & $\begin{array}{l}\text { - The self-exclusion program } \\
\text { facilitated positive results } \\
\text { including: reduced urges to } \\
\text { gamble, reduced DSM score, } \\
\text { reduced intensity of negative } \\
\text { consequences for gambling } \\
\text { (daily activities, social life, } \\
\text { work, mood) and an increased } \\
\text { perception of control } \\
\text { - At the 6-month follow-up, } \\
\text { more than half the participants } \\
\text { had breached their contract } \\
\text { or returned to a casino } \\
\text { - Self-exclusion detection } \\
\text { system needs to be improved }\end{array}$ \\
\hline $\begin{array}{l}\text { Lalande and Ladouceur } \\
\text { (2011) [63] }\end{array}$ & Limit-setting & $\begin{array}{l}\text { - Person sets a strict money } \\
\text { limit before beginning a } \\
\text { gambling session and an } \\
\text { intention to quit once } \\
\text { they reach the limit. }\end{array}$ & $\begin{array}{l}\text { - Both the PG and non-PG groups } \\
\text { use monetary loss limit as a form } \\
\text { of self-control to avoid overspending } \\
\text { - Those in the PG group set a higher } \\
\text { limit than those experiencing NPG; } \\
\text { those experiencing PG continue } \\
\text { spending after reaching their limit, } \\
\text { while those who were not engaged } \\
\text { in PG stop gambling } \\
\text { when reaching their limit } \\
\text { - Internal, external, implicit and } \\
\text { explicit limits are proposed to } \\
\text { operationalize self-control and } \\
\text { self-regulation } \\
\text { during gambling sessions }\end{array}$ \\
\hline $\begin{array}{l}\text { Linardatou et al. (2014) } \\
\text { [64] }\end{array}$ & $\begin{array}{l}\text { Stress management } \\
\text { - Relaxation } \\
\text { Breathing } \\
\text { - Progressive Muscle } \\
\text { Relaxation }\end{array}$ & $\begin{array}{l}\text { Relaxation Breathing: } \\
\text { - Individual engages in a } \\
\text { controlled method of } \\
\text { breathing to induce relaxation. } \\
\text { Progressive Muscle Relaxation: } \\
\text { - Individual engages in consecutive } \\
\text { contractions and relaxations of } \\
\text { different muscle groups } \\
\text { (skeletal, facial and respiratory) } \\
\text { in a down-top orientation } \\
\text { to induce relaxation. } \\
\text { - A CD guides them through } \\
\text { the relaxation techniques } \\
\text { (10 min RB, } 15 \text { min PMR); the } \\
\text { CD instructs them to perform } \\
\text { the techniques twice a day for } \\
8 \text { weeks at home } \\
\text { (maximum } 112 \text { sessions) } \\
\text { and to notice the difference } \\
\text { between being tense and relaxed } \\
\text { to improve perception } \\
\text { of the relaxation response. }\end{array}$ & $\begin{array}{l}\text { - The intervention group } \\
\text { demonstrated statistically } \\
\text { significant improvements in stress, } \\
\text { depression, anxiety symptoms, } \\
\text { life satisfaction and better daily routines } \\
\text { - Stress management may } \\
\text { provide psychosocial benefits } \\
\text { and improve the well-being of } \\
\text { individuals with pathological gambling } \\
\text { - It can be incorporated into PG programs }\end{array}$ \\
\hline $\begin{array}{l}\text { Luquiens et al. (2016) } \\
\text { [65] }\end{array}$ & $\begin{array}{l}\text { Self-help book } \\
\text { (Workbook) }\end{array}$ & $\begin{array}{l}\text { - The CBT workbook includes } \\
\text { content on motivation, } \\
\text { financial issues, cognitive } \\
\text { distortions, triggers, life } \\
\text { reorganization and } \\
\text { relapse prevention. }\end{array}$ & $\begin{array}{l}\text { - No significant difference in efficacy } \\
\text { between the group with guidance } \\
\text { compared to the group } \\
\text { without guidance and control group } \\
\text { - Internet-based CBT should } \\
\text { include intrinsic motivational } \\
\text { components to increase engagement }\end{array}$ \\
\hline
\end{tabular}


Table 2 Self-management strategies described in the included studies (Continued)

\begin{tabular}{|c|c|c|c|}
\hline Authors & $\begin{array}{l}\text { Self-Management } \\
\text { Strategy }\end{array}$ & $\begin{array}{l}\text { Details/ Description of } \\
\text { Self-Management Strategy }\end{array}$ & $\begin{array}{l}\text { Key Findings and Implementation } \\
\text { Considerations related to the } \\
\text { Self-Management Strategy }\end{array}$ \\
\hline Martin (2013) [66] & $\begin{array}{l}\text { Self-help online } \\
\text { toolkit }\end{array}$ & $\begin{array}{l}\text { - Toolkit is designed to help } \\
\text { individuals figure out if they } \\
\text { need to change their gambling } \\
\text { behaviour and decide how } \\
\text { to deal with the process of change. } \\
\text { - It contains exercises to help } \\
\text { determine the costs and benefits } \\
\text { of gambling and information } \\
\text { on managing urges. }\end{array}$ & $\begin{array}{l}\text {-When completed by a large number } \\
\text { of people, online health surveys } \\
\text { may be advantageous for screening, } \\
\text { intervening and providing self-help } \\
\text { information for disordered gambling }\end{array}$ \\
\hline \multirow[t]{4}{*}{ Moore et al. (2012) [67] } & \multirow[t]{4}{*}{$\begin{array}{l}\text { Various } \\
\text { - Cognitive } \\
\text { Approaches } \\
\text { - Direct Action } \\
\text { - Social Experience } \\
\text { - Avoidance } \\
\text { - Limit Setting }\end{array}$} & $\begin{array}{l}\text { Cognitive Approaches: } \\
\text { - Individual re-orders their priorities } \\
\text { with respect to gambling, thinks } \\
\text { about it differently and focuses } \\
\text { on different things. } \\
\text { Direct Action: } \\
\text { - Individual uses strategies such as } \\
\text { cutting up credit cards } \\
\text { or self-exclusion. }\end{array}$ & \multirow[t]{4}{*}{$\begin{array}{l}\text { - Problem gamblers who were } \\
\text { trying to reduce their gambling } \\
\text { were more likely to use strategies } \\
\text { such as Cognitive Approaches, } \\
\text { Direct Action, Social Experience, } \\
\text { Avoidance and Limit Setting } \\
\text { than other gambler groups }\end{array}$} \\
\hline & & $\begin{array}{l}\text { Social Experience: } \\
\text { - Individual maximizes the likelihood } \\
\text { that spending time at gambling } \\
\text { venues would be socially oriented } \\
\text { rather than gambling focused. }\end{array}$ & \\
\hline & & $\begin{array}{l}\text { Avoidance: } \\
\text { - Individual avoids gambling } \\
\text { venues and places personal } \\
\text { restrictions on their access } \\
\text { o money at venues. }\end{array}$ & \\
\hline & & $\begin{array}{l}\text { Limit Setting: } \\
\text { - Individual sets a strict money or time limit before beginning a gambling } \\
\text { session and quits gambling once they reach that limit. }\end{array}$ & \\
\hline Nelson et al. (2010) [68] & Self-exclusion & $\begin{array}{l}\text { - Individual enrolled in the } \\
\text { Missouri self-exclusion program } \\
\text { are responsible for not entering } \\
\text { any casinos in the state. }\end{array}$ & $\begin{array}{l}\text { - Enrolment in the self-exclusion } \\
\text { program reduced gambling } \\
\text { behaviour at } 6 \text { months }\end{array}$ \\
\hline $\begin{array}{l}\text { Subramaniam et al. } \\
\text { (2017) [69] }\end{array}$ & $\begin{array}{l}\text { Responsible } \\
\text { gambling strategies } \\
\text { - Delayed } \\
\text { Gratification } \\
\text { - Setting Limits } \\
\text { - Maintaining } \\
\text { Balance }\end{array}$ & $\begin{array}{l}\text { Delayed gratification: } \\
\text { - Individual quells the need for } \\
\text { immediate results. } \\
\text { Setting limits: } \\
\text { - Individual sets time or 'money' } \\
\text { limits on themselves. } \\
\text { Maintaining balance: } \\
\text { - Individual maintains a sense of } \\
\text { balance in terms of their } \\
\text { behaviour; curbs their excessive } \\
\text { spending, or time spent gambling. }\end{array}$ & $\begin{array}{l}\text { - The main theme of responsible } \\
\text { gambling was comprised of } \\
\text { two themes: self-development } \\
\text { strategies and limit gambling } \\
\text { related harm and family } \\
\text { interventions to reduce the } \\
\text { harm from gambling } \\
\text { - Subthemes included delayed } \\
\text { gratification, perception of futility } \\
\text { of gambling, settling limits, maintaining } \\
\text { balance, help-seeking and awareness } \\
\text { of disorders gambling in self or others } \\
\text { - Families play a significant role in } \\
\text { Asian societies in imposing RG, } \\
\text { education and counseling of } \\
\text { families is important }\end{array}$ \\
\hline $\begin{array}{l}\text { Toneatto et al. } \\
\text { (2014) [70] }\end{array}$ & $\begin{array}{l}\text { Workbook; } \\
\text { Mindfulness }\end{array}$ & $\begin{array}{l}\text {-Workbook contains CBT and } \\
\text { CD-guided mindfulness } \\
\text { (15-min mindfulness instruction } \\
\text { \& 30-min practice session). } \\
\text { - Mindfulness content contains } \\
\text { exercises and information } \\
\text { about awareness of breathing; } \\
\text { shifting thoughts of gambling; } \\
\text { being present-focused; awareness } \\
\text { of cognitive processes } \\
\text { (especially thoughts about gambling). } \\
\text { - CBT content contains instruction } \\
\text { in traditional content-focused } \\
\text { techniques, such as behavioural } \\
\text { problem-solving \& } \\
\text { cognitive restructuring. }\end{array}$ & $\begin{array}{l}\text { - Compared to a wait list control, } \\
\text { the mindfulness intervention } \\
\text { significantly reduced the severity } \\
\text { of gambling, gambling urges and } \\
\text { psychiatric symptoms at end-of treatment } \\
\text { - There was a significant decrease } \\
\text { in the proportion of the sample } \\
\text { meeting criteria for pathological gambling }\end{array}$ \\
\hline Townshend (2007) [71] & Self-exclusion & $\begin{array}{l}\text { Self-Exclusion in New Zealand } \\
\cdot \text { - Individual self-excludes themselves }\end{array}$ & $\begin{array}{l}\text { - Self-exclusion is an effective treatment } \\
\text { tool for participants who have an }\end{array}$ \\
\hline
\end{tabular}


Table 2 Self-management strategies described in the included studies (Continued)

\begin{tabular}{|c|c|c|c|}
\hline Authors & $\begin{array}{l}\text { Self-Management } \\
\text { Strategy }\end{array}$ & $\begin{array}{l}\text { Details/ Description of } \\
\text { Self-Management Strategy }\end{array}$ & $\begin{array}{l}\text { Key Findings and Implementation } \\
\text { Considerations related to the } \\
\text { Self-Management Strategy }\end{array}$ \\
\hline & & $\begin{array}{l}\text { from a gambling venue for a } \\
\text { period up to } 2 \text { years and there } \\
\text { may be a fine of } \$ 00 \text { if they } \\
\text { breach the self-exclusion ban. }\end{array}$ & \multirow{3}{*}{$\begin{array}{l}\text { "extreme difficulty" controlling } \\
\text { their gambling using other methods } \\
\text { - Self-exclusion may be more effective } \\
\text { in a jurisdiction with a public health } \\
\text { environment than has } \\
\text { been reported in other jurisdictions. }\end{array}$} \\
\hline & & $\begin{array}{l}\text { Self-Exclusion in the U.S. } \\
\text { - Regulations on self-exclusion } \\
\text { vary by state. }\end{array}$ & \\
\hline & & $\begin{array}{l}\text { Self-exclusion in Canada } \\
\text { (British Columbia): } \\
\text { - The British Columbia Lotteries } \\
\text { Corporation allows for individuals } \\
\text { to self-exclude for } 6 \text { months, } \\
1,2 \text { or } 3 \text { years and there may } \\
\text { be a fine of } \$ 5000 \text { if the } \\
\text { self-exclusion is violated } \\
\text { - This self-exclusion applies to } \\
\text { all venues with slot machines, } \\
\text { commercial bingo halls across } \\
\text { British Columbia and/or the } \\
\text { PlayNow locations. }\end{array}$ & \\
\hline
\end{tabular}

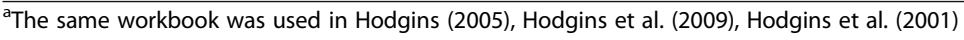

\section{Cognitive strategies}

Cognitive self-management strategies address thoughts, beliefs and cognitions surrounding gambling. Two studies described cognitive restructuring which involves changing irrational or negative thoughts and beliefs about gambling and replacing them with realistic and positive thoughts and beliefs to limit PG. Jauregui at al [59] reported no significant mediating effect of cognitive restructuring on anxiety between those experiencing pathological gambling and those who did not gamble. Moore et al. [67] examined use of cognitive restructuring for self-regulation of gambling among those with (PG) and without (NPG) gambling problems. They were interested in whether gambling status (PG and NPG) and frequency of gambling (low versus high) was associated with use of cognitive restructuring. They found that the PG-high frequency group was most likely to use cognitive restructuring, followed by PG-low frequency, NPG-high frequency, and NPG-low frequency.

\section{Coping strategies}

Four studies described self-management strategies in the form of coping skills and/or self-directed activities to improve coping skills. Both adaptive and maladaptive strategies were described including mindfulness) [70], emotional expression [59], relaxation breathing [64], progressive muscle relaxation [64], social support [59], problem solving [59], avoidance [59, 67], wishful thinking [59], social withdrawal [59], self-criticism [59], and imaginal desensitization ${ }^{1}$ [48]. Mindfulness and imaginal desensitization reduced gambling severity and gambling urges among a population of people experiencing PG $[48,70]$. Maladaptive coping strategies such as avoidance, wishful thinking, social withdrawal, self-criticism, and emotional expression were associated with higher PG scores [59]. In one study, relaxation breathing and progressive muscle relaxation were effective strategies in reducing stress, depression and anxiety, and improving life satisfaction and daily routines (e.g., breakfast and dinner) among people experiencing PG [64].

\section{Multi-part interventions}

Multi-part self-management interventions provide a variety of tools to help people who want to change their gambling behavior to monitor their gambling activities, set and monitor goals, use self-reflection to recognize underlying motivations and repercussion of their addiction. These interventions include the use of workbooks, self-directed CBT interventions, self-help toolkits, booklets, and personalized feedback tools.

Self-directed CBT Two studies described online CBT interventions for use without the assistance of a therapist $[44,45]$ such as challenging and replacing erroneous thoughts. They also contained other self-management strategies such as debt management, managing high risk situations, recognizing triggers $[44,45]$, imaginal desensitization, relaxation training, goal setting, emotions maintenance, relapse prevention [44], psychoeducation, and identifying social consequences of gambling [45]. Casey et al. [44] found that a CBT intervention was associated with reduced gambling severity, other PG and mental health outcomes, and greater life satisfaction after the initial treatment and at 12-months follow-up. The CBT intervention in a study by Castrén et al. [45] was associated with reduced gambling-related problems, urges, impaired control of gambling, social 
consequences, gambling-related cognitive erroneous thoughts and depression.

Workbooks Nine studies examined online and offline workbooks with exercises meant to manage PG-related outcomes. Although the structure of the workbooks and the topics varied, common elements included motivation to change and the change process [42, 43, 72] and self-reflection and improved self-awareness of gambling related cognitions [43, 70]. Most workbooks contained some CBT content, such as information on cognitive distortions and cognitive- restructuring $[43,54-56,58$, 70]. Others included materials and exercises on goal-setting [42] and finances [65]. Most workbooks provided descriptions and information for other self-management strategies noted in this review such as mindfulness [42, 70], limiting strategies [43], self-exclusion [54-56], stress management [42] and alternative activity scheduling [54-56]. The majority of workbooks included information relating to managing urges and/or relapses, maintenance and resources [42, $43,54-56,58,65]$. Some studies suggested that workbook-only interventions were effective in reducing harms associated with PG [42, 54] while other studies noted improved outcomes when the workbook was paired with therapist guidance or other formal support $[43,55,58]$. Generally, the workbook interventions were reported to be well-received by clients and described as an approach to expand PG services to individuals.

Booklets and toolkits Informational booklet [57] and self-help toolkit $[60,66]$ interventions are similar in structure and content to workbook interventions and were utilized in three studies. Like workbooks, both toolkits and the informational booklets contained resources on managing urges, the change process and relapse prevention. The primary focus of the toolkits was to help individuals self-reflect on their gambling behaviours and included exercises to determine the costs and benefits of gambling behaviour to motivate change. The booklets included additional information on lifestyle balance, financial issues and managing comorbid conditions (e.g., emotional and addiction problems). LaBrie et al. [60] reported improved PG-related outcomes for toolkit recipients and advised that toolkits may be a viable treatment alternative for individuals who do not want to engage in formal treatment courses. Hodgins et al. [57] reported that participants who received repeated mailing of bibliotherapy (relapse prevention booklets) for PG were more likely to meet their gambling-related treatment goals than those who did not receive mailings. However, participants who received repeated mailings did not differ from participants who received a single mailing on gambling frequency or reported gambling losses.

Personalized feedback tool Online personalized feedback tools were noted in two studies [46, 47]. Personalized feedback tools involve some form of self-assessment of PG behaviour and/or ongoing information gathering to provide a personalized profile of PG behaviour, beliefs and habits. This information is then presented back to the user along with a comparison of their behaviour with the gambling behaviour or cognitions of other users and/or general population to establish a risk level for PG. This feedback is presented along with helpful strategies and techniques to lower risk and limit gambling. Additionally, in cases of continual information gathering/behaviour tracking, individuals could opt-in to have personalized messages sent to them when they were engaging in risky gambling behaviours. Cunningham et al. [46] reported a reduction in the number of days gambled for participants receiving a partial feedback tool (i.e., feedback about behaviour without comparison to norm for that behaviour) in comparison to those who received no intervention. They found no evidence to support the efficacy of a normative feedback tool. Forsström et al. [47] found low continued usage of a personalized feedback tool among participants despite positive opinions of its content.

\section{Discussion}

The purpose of this paper was to examine the scope of current published literature on PG self-management strategies. We identified 31 studies and reviewed 23 different self-management strategies for PG, published between 2000 and 2017. In a previous review of self-help for PG (up to April 2008) only two types of self-help had been reported; these were self-help manuals and audiotapes [35]. Our findings show that there is a growing body of literature examining a diverse range of self-management strategies for PG. The most commonly cited strategies in this review were self-exclusion $(n=9)$, workbooks $(n=8)$, and money or time limiting strategies $(n=4)$. Other strategies included various cognitive and behavioural strategies, coping strategies, stress management, and mindfulness. Surprisingly, technological modes of treatment (e.g., virtual reality treatments) were not well-represented in this review. Three studies examining the use of CBT in virtual reality with therapist assistance (therapist-assisted studies were excluded from our review), showed that the technology has promise for the treatment of PG [73]. Although the self-management strategies noted in this review are conceptually similar to those identified in other literature [35-37], the labelling and categorization of strategies was found to considerably vary across studies. Future research examining 
self-management strategies for PG would benefit from standardized conceptualizations of strategies and shared terminology.

Self-exclusion was the most examined approach to self-management in this review, yet there is little evidence for its effectiveness. In fact, compliance rates are quite low (13 to $30 \%$ ), with inadequate surveillance and enforcement of bans, and complicated enrollment processes which impede use of this option to manage PG $[51,74]$. A deeper understanding of self-exclusion, in particular, and other self-management strategies requires consideration of comorbid health and social concerns $[6$, 75]. People who experience complex health and social concerns such as homelessness, mental health issues, substance use disorders, and incarceration are at greater risk of PG, yet current services do not address multimorbidity $[3,4]$. Notably, although some studies in this review included participants with mental health and/or substance use comorbidities, most did not explicitly address these comorbidities with PG (e.g., alcoholism) in the design of self-management strategies. Further research is needed to explore the complex interplay between PG and comorbid conditions and design comprehensive interventions that address multiple needs $[6,75]$.

In addition, a key finding from this review was a lack of research examining self-management approaches tailored to specific socio-demographic sub-groups (e.g., age, income, gender, ethnicity, geography) [49]. There were few studies among younger populations aged 18 to 35 years old. Only one study focused on older adults who were aged 60 and above [69]. Older adults have their own unique concerns that may affect how they use self-management strategies and the types of strategies that they prefer (e.g., access to and familiarity with technology) [76]. Only one study examined participants with low-income backgrounds [43]. While many studies had mixed-sex samples only one study considered a gendered approach to self-management strategies. One study reported that, within Asian culture, families use a variety of mechanisms to enforce responsible gambling (e.g., family exclusion orders) [69]. Research is needed to understand what self-management approaches may be appropriate and effective for a variety of populations.

Many empirically validated theories of behaviour change, including diffusion of innovations [77], social cognitive theory [78], and the social ecological model [79] assert that social relationships play a significant role in facilitating behavioural change. These findings suggest that we need a better understanding of the role that support networks/circles, and peer support outside the formal treatment environment may play in PG self-management $[76,80]$.This topic deserves specific exploration of those strategies that may or may not be effective for specific populations, such as people facing poverty and homelessness and those from varying ethnic cultures. This could be accomplished using a realist perspective to understand what works, for whom, and under what conditions [81, 82]. Given the majority of people experiencing PG do not actively seek treatment [13, 14], offering evidence of the effectiveness of personal approaches to self-management is imperative. Moreover, past research suggests that one-third to upwards of $82 \%$ of people experience natural recovery from PG with men more likely to report this happening than women $[19,83,84]$. While the literature on PG self-management strategies has evolved since Raylu et al's paper [35], there is still little evidence of the effectiveness of self-management to reduce harms associated with gambling.

\section{Strengths and limitations}

This study has a few limitations that should be noted. It is also possible that some relevant articles were missed, as only articles published between January 1, 2000 and June 28, 2017 were included as well as those published in English. We included French publications in our initial search with the idea that we would review English translations of papers written in French, but no translations were available. None of the authors are proficient in French language. However, our search strategy was comprehensive and guided by an information science specialist. To our knowledge, this is the first review of self-management strategies for PG since a previous review in 2008. The earlier review focused on broad definition of self-help that included forming partnerships with heath care providers [25]. We defined self-management with a narrower scope focusing on self-care outside the health and social service system as we were specifically interested in strategies that people manage on their own (or after active treatment) to reduce personal harms associated with gambling. Our review focused on adults; however, PG among teens and young adults is a serious public health concern. As such it would be prudent to similarly explore self-management among those under age $18[85,86]$. This is especially important given that gambling seems to differ on a variety of dimensions among youth compared to adults, including reasons for gambling, comorbidities, and consequences of gambling [85, 87-90]. Individual capacity is an important consideration in any approach to care for gambling concerns, whether through professional treatment or self-care. This becomes complicated by cognitive and intellectual disabilities, multimorbidities, and such social determinants of health as homelessness and poverty. In particular self-management may not be an appropriate approach to care. 


\section{Conclusions}

Given that it is the minority of people with gambling concerns that seek treatment, that stigma is an enormous barrier to care, that PG services are scarce and do not address multimorbidity [5, 6, 14, 91, 92], it is imperative that we examine the personal management of gambling as an option to formalized treatment. This is the first review to examine self-management of PG and findings indicate that evidence is lacking on this topic. It is imperative that the field explore self-management in PG in more depth and for specialized populations to understand the nuances of recovery for diverse populations.

\section{Endnotes}

${ }^{1}$ Imaginal desensitization is an approach through which patients learn to visualize a situation that triggers an impulse to carry out an impulsive behavior (e.g., gambling), and then imagine overcoming the urge using learned relaxation skills. The idea is that with time, an individual gains confidence in their ability to manage urges and becomes "desensitized" to triggering situations.

\section{Additional files}

Additional file 1: PRISMA-SCR Checklist (DOCX $271 \mathrm{~kb}$ )

Additional file 2: Medline Search Strategy (DOCX $15 \mathrm{~kb}$ )

\section{Abbreviations}

CBT: Cognitive Behavioural Therapy; EGM: CD: Compact Disk; IDMI: Imaginal Desensitization plus Motivational Interviewing; M: Mean; N: Number; NPG: Non-problem gambling; PG: Problem Gambling; PGSI: qProblem Gambling Severity Index; SD: Standard Deviation

\section{Acknowledgements}

We wish to thank Guido Tacchini and Natalie Waldbrook for their contributions to the paper.

\section{Funding}

The Centre for Urban Health Solutions is part of the Li Ka Shing Knowledge Institute of St. Michael's Hospital. This study was funded by the Province of Ontario (Province), Ministry of Health and Long-Term Care (Ministry Grant \#438). SJTG is supported by the Canadian Institutes for Health Research (CIHR) Embedded Clinician Scientist Award in Transitions in Care (20162020). The study was also supported by the Dalla Lana School of Public Health, University of Toronto The views expressed in this publication are the views of the authors and do not necessarily reflect those of the Province or the $\mathrm{CIHR}$.

\section{Availability of data and materials}

The authors confirm that the data supporting the findings of this study are available within the article.

\section{Authors' contributions}

FIM, SJTG, SHW designed the original project; FIM, SJTG, SHW, DTK, JLW designed and operationalized the specific research question and methodology for this review; CZ, DTK and JLW conducted the literature searches; DTK, JLW, FIM, SJTG, SHW, LC selected the papers for review and reviewed the final papers; DTK, JLW wrote the first draft of the paper; FIM, SHW, DTK, JLW, LC, CZ, SWH, SJTG revised and finalized the paper. All authors read and approved the final manuscript.
Ethics approval and consent to participate

Not applicable.

\section{Consent for publication}

Not applicable.

\section{Competing interests}

The authors declare that they have no competing interests.

\section{Publisher's Note}

Springer Nature remains neutral with regard to jurisdictional claims in published maps and institutional affiliations.

\section{Author details}

${ }^{1}$ MAP Centre for Urban Health Solutions, St. Michael's Hospital, 30 Bond Street, Toronto, ON M5B 1W8, Canada. ${ }^{2}$ Health Information Sciences Library, University of Toronto, Toronto, ON, Canada. ${ }^{3}$ Leslie Dan Faculty of Pharmacy, University of Toronto, Toronto, ON, Canada.

Received: 6 December 2018 Accepted: 8 April 2019

Published online: 29 April 2019

\section{References}

1. Calado F, Griffiths MD. Problem gambling worldwide: an update and systematic review of empirical research (2000-2015). J Behav Addict. 2016; 5(4):592-613.

2. Stucki S, Rihs-Middel M. Prevalence of adult problem and pathological gambling between 2000 and 2005: an update. J Gambl Stud. 2007;23(3): 245-57.

3. Matheson FI, Devotta K, Wendaferew A, Pedersen C. Prevalence of gambling problems among the clients of a Toronto homeless shelter. J Gambl Stud. 2014;30(2):537-46.

4. Cowlishaw S, Merkouris S, Chapman A, Radermacher H. Pathological and problem gambling in substance use treatment: a systematic review and meta-analysis. J Subst Abus Treat. 2014;46(2):98-105.

5. Ferentzy P, Wayne Skinner WJ, Matheson Fl. Illicit drug use and problem gambling. ISRN Addict. 2013;2013:342392.

6. Guilcher SJ, Hamilton-Wright S, Skinner W, Woodhall-Melnik J, Ferentzy P, Wendaferew A, Hwang SW. Matheson FI: "talk with me": perspectives on services for men with problem gambling and housing instability. BMC Health Serv Res. 2016;16(1):340.

7. Rush B, Moxam R.: Treatment of problem gambling in Ontario: service utilization and client characteristics, January 1, 1998, to April 30, 2000. DATIS report to the Ontario Ministry of Health and Long-Term Care. Toronto: Ontario Ministry of Health and Long-Term Care; 2001.

8. Toneatto T, Ladoceur R. Treatment of pathological gambling: a critical review of the literature. Psychol Addict Behav. 2003;17(4):284.

9. Hodgins DC, Holub A. Treatment of problem gambling. Research and measurement issues in gambling studies; 2007. p. 372-91.

10. Dowling NA, Cowlishaw S, Jackson AC, Merkouris SS, Francis KL, Christensen DR. Prevalence of psychiatric co-morbidity in treatment-seeking problem gamblers: a systematic review and meta-analysis. Australian \& New Zealand Journal of Psychiatry. 2015;49(6):519-39.

11. Turner NE, Preston DL, McAvoy S, Gillam L. Problem gambling inside and out: the assessment of community and institutional problem gambling in the Canadian correctional system. J Gambl Stud. 2013;29(3):435-51.

12. Rockloff MJ, Schofield G. Factor analysis of barriers to treatment for problem gambling. J Gambl Stud. 2004;20(2):121-6.

13. Suurvali H, Hodgins D, Toneatto $T$, Cunningham J. Treatment seeking among Ontario problem gamblers: results of a population survey. Psychiatr Serv. 2008;59(11):1343-6.

14. Cunningham JA, Breslin FC. Only one in three people with alcohol abuse or dependence ever seek treatment. Addict Behav. 2004;29(1):221-3.

15. Suurvali H, Cordingley J, Hodgins DC, Cunningham J. Barriers to seeking help for gambling problems: a review of the empirical literature. J Gambl Stud. 2009;25(3):407-24.

16. Goslar M, Leibetseder M, Muench HM, Hofmann SG, Laireiter A-R. Efficacy of face-to-face versus self-guided treatments for disordered gambling: a metaanalysis. J Behav Addict. 2017;6(2):142-62.

17. Dabrowska K, Moskalewicz J, Wieczorek $Ł$. Barriers in access to the treatment for people with gambling disorders. Are they different from 
those experienced by people with alcohol and/or drug dependence? J Gambl Stud. 2017;33(2):487-503.

18. Gainsbury S, Hing N, Suhonen N. Professional help-seeking for gambling problems: awareness, barriers and motivators for treatment. J Gambl Stud. 2014;30(2):503-19.

19. Hodgins DC, El-Guebaly N. Natural and treatment-assisted recovery from gambling problems: a comparison of resolved and active gamblers. Addiction. 2000;95(5):777-89.

20. Tavares H, Martins SS, Zilberman ML, el-Guebaly N. gamblers seeking treatment: why haven't they come earlier? Addict Disord Treat. 2002;1(2):65-9.

21. Pulford J, Bellringer M, Abbott M, Clarke D, Hodgins D, Williams J. Barriers to help-seeking for a gambling problem: the experiences of gamblers who have sought specialist assistance and the perceptions of those who have not. J Gambl Stud. 2009;25(1):33-48.

22. Taylor SJ, Pinnock H, Epiphaniou E, Pearce G, Parke HL, Schwappach A, Purushotham N, Jacob S, Griffiths CJ, Greenhalgh T. A rapid synthesis of the evidence on interventions supporting self-management for people with long-term conditions: PRISMS-practical systematic review of selfmanagement support for long-term conditions; 2014.

23. Barlow J, Wright C, Sheasby J, Turner A, Hainsworth J. Self-management approaches for people with chronic conditions. Patient Educ Couns. 2002; 48(2):177-87.

24. Center for the Advancement of Health. Essential elements of selfmanagement interventions. Washington, DC: Center for the Advancement of Health 2002.

25. Lorig KR, Holman HR. Self-management education: history, definition, outcomes, and mechanisms. Ann Behav Med. 2003;26(1):1-7.

26. Solomon MR. Information technology to support self-management in chronic care. Dis Manag Health Out. 2008;16(6):391-401.

27. Cafazzo JA, Casselman M, Hamming N, Katzman DK, Palmert MR. Design of an mHealth app for the self-management of adolescent type 1 diabetes: a pilot study. J Med Internet Res. 2012;14(3):e70. https://doi.org/10.2196/jmir. 2058.

28. Barrio P, Ortega L, López H, Gual A. Self-management and shared decisionmaking in alcohol dependence via a mobile app: a pilot study. Int J Behav Med. 2017;24(5):722-7.

29. Quanbeck A, Chih M-Y, Isham A, Johnson R, Gustafson D. Mobile delivery of treatment for alcohol use disorders: a review of the literature. Alcohol Res. 2014;36(1):111

30. Sakakibara BM, Kim AJ, Eng JJ. A systematic review and meta-analysis on self-management for improving risk factor control in stroke patients. Int J Behav Med. 2017;24(1):42-53.

31. Monney G, Penzenstadler L, Dupraz O, Etter J-F, Khazaal Y. mHealth app for cannabis users: satisfaction and perceived usefulness. Front Psych. 2015;6:120

32. Mains JA, Scogin FR. The effectiveness of self-administered treatments: a practice-friendly review of the research. J Clin Psychol. 2003;59(2):237-46.

33. Akın A, Arslan S, Arslan N, Uysal R, Sahranç Ü. Self-control Management and Internet Addiction. Int Online J Educ Sci. 2015;7(3):95-100.

34. Whiteman KL, Naslund JA, DiNapoli EA, Bruce ML, Bartels SJ. Systematic review of integrated general medical and psychiatric self-management interventions for adults with serious mental illness. Psychiatr Serv. 2016; 67(11):1213-25.

35. Raylu N, Oei TP, Loo J. The current status and future direction of self-help treatments for problem gamblers. Clin Psychol Rev. 2008;28(8):1372-85.

36. Rodda SN, Hing N, Hodgins DC, Cheetham A, Dickins M, Lubman DI. Change strategies and associated implementation challenges: an analysis of online Counselling sessions. J Gambl Stud. 2017;33(3):955-73.

37. Rodda SN, Bagot KL, Cheetham A, Hodgins DC, Hing N, Lubman DI. Types of change strategies for limiting or reducing gambling behaviors and their perceived helpfulness: a factor analysis. Psychol Addict Behav. 2018;32(6):679-88.

38. Arksey H, O'Malley L. Scoping studies: towards a methodological framework. Int J Soc Res Methodol. 2005;8(1):19-32.

39. Moher D, Shamseer L, Clarke M, Ghersi D, Liberati A, Petticrew M, Shekelle P, Stewart LA. Preferred reporting items for systematic review and meta-analysis protocols (PRISMA-P) 2015 statement. Systematic reviews. 2015;4(1):1

40. Tricco AC, Lillie E, Zarin W, O'Brien KK, Colquhoun H, Levac D, Moher D, Peters MDJ, Horsley T, Weeks L, et al. PRISMA extension for scoping reviews (PRISMAScR): checklist and explanation. Ann Intern Med. 2018;169(7):467-73.

41. Avery L, Davis DR. Women's recovery from compulsive gambling: formal and informal supports. J Soc Work Pract Addict. 2008;8(2):171-91.
42. Boughton RR, Jindani F, Turner NE. Group treatment for women gamblers using web, teleconference and workbook: effectiveness pilot. Int J Ment Heal Addict. 2016;14(6):1074-95.

43. Campos MD, Rosenthal RJ, Chen Q, Moghaddam J, Fong TW. A self-help manual for problem gamblers: the impact of minimal therapist guidance on outcome. Int J Ment Heal Addict. 2016;14(4):579-96.

44. Casey LM, Oei TPS, Raylu N, Horrigan K, Day J, Ireland M, Clough BA. Internet-based delivery of cognitive behaviour therapy compared to monitoring, feedback and support for problem gambling: a randomised controlled trial. J Gambl Stud. 2017;33(3):993-1010.

45. Castrén S, Pankakoski M, Tamminen M, Lipsanen J, Ladouceur R, Lahti T. Internet-based CBT intervention for gamblers in Finland: experiences from the field. Scand J Psychol. 2013:54(3):230-5.

46. Cunningham JA, Hodgins DC, Toneatto T, Murphy M. A Randomized Controlled Trial of a Personalized Feedback Intervention for Problem Gamblers. PLoS One. 2012;7(2)

47. Forsström D, Jansson-Fröjmark $M$, Hesser $H$, Carlbring P. Experiences of Playscan: interviews with users of a responsible gambling tool. Internet Interv. 2017:8:53-62.

48. Grant JE, Donahue CB, Odlaug BL, Kim SW. A 6-month follow-up of imaginal desensitization plus motivational interviewing in the treatment of pathological gambling. Ann Clin Psychiatry. 2011;23(1):3-10.

49. Hayer T, Meyer G. Self-exclusion as a harm minimization strategy: evidence for the casino sector from selected European countries. J Gambl Stud. 2011; 27(4):685-700

50. Hing N, Russell AMT, Gainsbury SM, Blaszczynski A. Characteristics and helpseeking behaviors of internet gamblers based on most problematic mode of gambling. J Med Internet Res. 2015;17(1).

51. Hing N, Sproston K, Tran K, Russell AM. Gambling responsibly: who does it and to what end? J Gambl Stud. 2017:33(1):149-65.

52. Hing N, Cherney L, Gainsbury SM, Lubman DI, Wood RT, Blaszczynski A. Maintaining and losing control during internet gambling: a qualitative study of gamblers' experiences. New Media Soc. 2015;17(7):1075-95.

53. Hing N, Russell A, Tolchard B, Nuske E. Are there distinctive outcomes from selfexclusion? An exploratory study comparing gamblers who have self-excluded, received Counselling, or both. Int J Ment Heal Addict. 2015;13(4):481-96.

54. Hodgins DC. Implications of a brief intervention trial for problem gambling for future outcome research. J Gambl Stud. 2005;21(1):13-9.

55. Hodgins DC, Currie SR, Currie G, Fick GH. Randomized trial of brief motivational treatments for pathological gamblers: more is not necessarily better. J Consult Clin Psychol. 2009;77(5):950.

56. Hodgins DC, Currie SR, el-Guebaly N. Motivational enhancement and selfhelp treatments for problem gambling. J Consult Clin Psychol. 2001;69(1):50.

57. Hodgins DC, Currie SR, el-Guebaly N, Diskin KM. Does providing extended relapse prevention Bibliotherapy to problem gamblers improve outcome? J Gambl Stud. 2007;23(1):41-54.

58. Hodgins DC, Currie S, el-Guebaly N, Peden N. Brief motivational treatment for problem gambling: a 24-month follow-up. Psychol Addict Behav. 2004; 18(3):293.

59. Jauregui P, Onaindia J, Estévez A. Adaptive and maladaptive coping strategies in adult pathological gamblers and their mediating role with anxious-depressive symptomatology. J Gambl Stud. 2017;33(4):1081-97.

60. LaBrie RA, Peller AJ, LaPlante DA, Bernhard B, Harper A, Schrier T, Shaffer HJ. A brief self-help toolkit intervention for gambling problems: a randomized multisite trial. Am J Orthopsychiatry. 2012;82(2):278-89.

61. Ladouceur R, Jacques C, Giroux I, Ferland F, Leblond J. Brief communications analysis of a Casino's self-exclusion program. J Gambl Stud. 2000;16(4):453-60.

62. Ladouceur R, Sylvain C, Gosselin P. Self-exclusion program: a longitudinal evaluation study. J Gambl Stud. 2007;23(1):85-94.

63. Lalande DR, Ladouceur R. Can cybernetics inspire gambling research? A limitbased conceptualization of self-control. Int Gambl Stud. 2011;11(2):237-52.

64. Linardatou C, Parios A, Varvogli L, Chrousos G, Darviri C. An 8-week stress management program in pathological gamblers: a pilot randomized controlled trial. J Psychiatr Res. 2014;56:137-43.

65. Luquiens A, Tanguy M, Lagadec M, Benyamina A, Aubin H, Reynaud M. The Efficacy of Three Modalities of Internet-Based Psychotherapy for NonTreatment-Seeking Online Problem Gamblers: A Randomized Controlled Trial. J Med Internet Res. 2016;18(2).

66. Martin RJ. The feasibility of providing gambling-related self-help information to college students who screen for disordered gambling via an online health survey: an exploratory study. J Gambl Issues. 2013;28:1-8. 
67. Moore S, Thomas AC, Kyrios M, Bates G. The self-regulation of gambling. J Gambl Stud. 2012;28(3):405-20.

68. Nelson SE, Kleschinsky JH, LaBrie RA, Kaplan S, Shaffer HJ. One decade of self exclusion: Missouri casino self-excluders four to ten years after enrollment. J Gambl Stud. 2010;26(1):129-44.

69. Subramaniam M, Satghare P, Vaingankar JA, Picco L, Browning CJ, Chong S, Thomas SA. Responsible gambling among older adults: a qualitative exploration. BMC Psychiatry. 2017;17(1):124.

70. Toneatto T, Pillai S, Courtice E. Mindfulness-enhanced cognitive behavior therapy for problem gambling: a controlled pilot study. Int J Ment Heal Addict. 2014;12(2):197-205.

71. Townshend P. Self-exclusion in a public health environment: an effective treatment option in New Zealand. Int J Ment Heal Addict. 2007;5(4):390-5.

72. Luquiens $\mathrm{A}$, Lagadec M, Tanguy M, Reynaud M. Efficacy of online psychotherapies in poker gambling disorder: an online randomized clinical trial. Eur Psychiatry. 2015;30:1053.

73. Bouchard S, Robillard G, Giroux I, Jacques C, Loranger C, St-Pierre M, Chrétien M, Goulet A. Using virtual reality in the treatment of gambling disorder: the development of a new tool for cognitive behavior therapy. Front Psych. 2017:8:27.

74. Motka F, Grune B, Sleczka P, Braun B, Ornberg JC, Kraus L. Who uses selfexclusion to regulate problem gambling? A systematic literature review. J Behav Addict. 2018:1-14.

75. Kotter R, Kraplin A, Pittig A, Buhringer G. A systematic review of land-based self-exclusion programs: demographics, Gambling Behavior, Gambling Problems, Mental Symptoms, and Mental Health. J Gambl Stud. 2018. https://doi.org/10.1007/s10899-018-9777-8.

76. Matheson Fl, Sztainert T, Lakman Y, Steele SJ, Ziegler CP, Ferentzy P. Prevention and Treatment of Problem Gambling Among Older Adults: a scoping review. J GAmbl Issues. 2018;39:6-66.

77. Rogers EM. Diffusion of innovations: Simon and Schuster; 2010.

78. Bandura A. Social cognitive theory. Handbook of social psychological theories. 2012;2011:349-73.

79. Sallis JF, Owen N, Fisher E. Ecological models of health behavior. Health behavior: Theory, research, and practice. 2015;5:43-64.

80. Rash CJ, Petry NM. Psychological treatments for gambling disorder. Psychol Res Behav Manag. 2014;7:285-95.

81. Fitzpatrick S. Explaining Homelessness: a Critical Realist Perspective. Housing Theory and Society. 2005;(1):1, 17.

82. Vassilev I, Rogers A, Sanders C, Kennedy A, Blickem C, Protheroe J, Bower P, Kirk S, Chew-Graham C, Morris R. Social networks, social capital and chronic illness self-management: a realist review. Chronic IIIn. 2011;7(1):60-86.

83. Slutske WS. Natural recovery and treatment-seeking in pathological gambling: results of two U.S. national surveys. Am J Psychiatry. 2006;163(2):297-302.

84. Slutske WS, Blaszczynski A, Martin NG. Sex differences in the rates of recovery, treatment-seeking, and natural recovery in pathological gambling: results from an Australian community-based twin survey. Twin Res Hum Genet. 2009;12(5):425-32.

85. Kryszajtys DT, Hahmann TE, Schuler A, Hamilton-Wright S, Ziegler CP, Matheson Fl. Problem gambling and delinquent behaviours among adolescents: a scoping review. J Gambl Stud. 2018:1-22.

86. Neighbors C, Rodriguez LM, Rinker DV, Gonzales RG, Agana M, Tackett JL, Foster DW. Efficacy of personalized normative feedback as a brief intervention for college student gambling: a randomized controlled trial. J Consult Clin Psychol. 2015;83(3):500.

87. Delfabbro $P$, Thrupp $L$. The social determinants of youth gambling in south Australian adolescents. J Adolesc. 2003;26(3):313-30.

88. Hardoon KK, Gupta R, Derevensky JL. Psychosocial variables associated with adolescent gambling. Psychol Addict Behav. 2004;18(2):170-9.

89. Lussier ID, Derevensky J, Gupta R, Vitaro F. Risk, compensatory, protective, and vulnerability factors related to youth gambling problems. Psychol Addict Behav. 2014;28(2):404-13.

90. Martins SS, Liu W, Hedden SL, Goldweber A, Storr CL, Derevensky JL, Stinchfield R, lalongo NS, Petras H. Youth aggressive/disruptive behavior trajectories and subsequent gambling among urban male youth. J Clin Child Adolesc Psychol. 2013;42(5):657-68.

91. Baxter A, Salmon C, Dufresne K, Carasco-Lee A, Matheson Fl. Gender differences in felt stigma and barriers to help-seeking for problem gambling. Addict Behav Rep. 2016;3:1-8.

92. Hing N, Russell AM. How anticipated and experienced stigma can contribute to self-stigma: the case of problem gambling. Front Psychol. 2017;8:235.

Ready to submit your research? Choose BMC and benefit from:

- fast, convenient online submission

- thorough peer review by experienced researchers in your field

- rapid publication on acceptance

- support for research data, including large and complex data types

- gold Open Access which fosters wider collaboration and increased citations

- maximum visibility for your research: over $100 \mathrm{M}$ website views per year

At BMC, research is always in progress.

Learn more biomedcentral.com/submissions 